\title{
Topology optimization of compliant mechanisms with stress constraints and manufacturing error robustness
}

\author{
Assis da Silva, Gustavo; Teófilo Becka, André ; Sigmund, Ole
}

Published in:

Computer Methods in Applied Mechanics and Engineering

Link to article, DOI:

10.1016/j.cma.2019.05.046

Publication date:

2019

Document Version

Peer reviewed version

Link back to DTU Orbit

Citation (APA):

Assis da Silva, G., Teófilo Becka, A., \& Sigmund, O. (2019). Topology optimization of compliant mechanisms with stress constraints and manufacturing error robustness. Computer Methods in Applied Mechanics and Engineering, 354, 397-421. https://doi.org/10.1016/j.cma.2019.05.046

\section{General rights}

Copyright and moral rights for the publications made accessible in the public portal are retained by the authors and/or other copyright owners and it is a condition of accessing publications that users recognise and abide by the legal requirements associated with these rights.

- Users may download and print one copy of any publication from the public portal for the purpose of private study or research.

- You may not further distribute the material or use it for any profit-making activity or commercial gain

- You may freely distribute the URL identifying the publication in the public portal 


\title{
Topology optimization of compliant mechanisms with stress constraints and manufacturing error robustness
}

\author{
Gustavo Assis da Silva ${ }^{\mathrm{a}}$, André Teófilo Beck ${ }^{\mathrm{a}}$, Ole Sigmund ${ }^{\mathrm{b}}$ \\ ${ }^{a}$ Department of Structural Engineering, São Carlos School of Engineering, University of São Paulo, 13.566-590, São Carlos, SP, Brazil \\ ${ }^{b}$ Department of Mechanical Engineering, Solid Mechanics, Technical University of Denmark, Nils Koppels Alle, B. 404,2800 Kgs. Lyngby, Denmark
}

\begin{abstract}
This work proposes a robust formulation to address the compliant mechanism design problem subject to both stress constraints and manufacturing uncertainty. The proposed formulation is an extension of the robust approach for compliant mechanism design based on eroded, intermediate and dilated projections. The novelty in this proposal comes from inclusion of a stress failure criterion in each projected field, in order to ensure compliant mechanisms that satisfy the stress failure criterion even in the presence of uniform manufacturing variations. The objective of the optimization problem is the minimization of the maximum displacement at the output port of the mechanism, given eroded, intermediate and dilated designs, subjected to upper and lower volume constraints and one stress constraint per finite element on each of the three projected fields. The objective function is weighted by the volume of the dilated topology, in order to avoid possible numerical instabilities that may occur when the upper volume constraint is not active. Several examples are solved and the optimized results are post-processed with body-fitted finite element meshes. Numerical results demonstrate that: 1) the proposed stress-constrained robust approach provides results in which both maximum stress and output displacements are robust with respect to uniform boundary variations; however, while the maximum stress is almost insensitive to manufacturing variations, the output displacement does show some degradation when compared with the traditional robust approach; 2) the traditional robust approach, i.e., without the stress considerations, provides results in which the maximum stress has unpredictable and non-smooth behavior after uniform boundary variation; and 3) the stress-constrained deterministic approach, i.e., without considering the manufacturing uncertainty, provides results in which both maximum stress and output displacements are non-robust with respect to uniform boundary variations.
\end{abstract}

Keywords: Topology optimization, Robust design, Compliant mechanism, Manufacturing uncertainty, Stress constraint

\section{Introduction}

This paper deals with two important topics in compliant mechanisms design through topology optimization: 1) stress constraints; and 2) manufacturing uncertainty. These are topics that often arise when trying to satisfy realistic engineering requirements during the topology design of compliant mechanisms.

Handling stress constraints in topology optimization of compliant mechanisms is a challenging task. The main difficulties associated with the traditional problem of volume minimization with stress constraints, that are the large number of local stress constraints and the singularity phenomenon [1], also affect the compliant mechanism design problem [2]. Moreover, the compliant mechanism design problem is kinematically more complex than the volume

Email addresses: gustavoas@usp.br (Gustavo Assis da Silva), atbeck@sc.usp.br (André Teófilo Beck), sigmund@mek.dtu.dk (Ole Sigmund) 
minimization problem. Despite these difficulties, the stress-constrained design problem of compliant mechanism has been studied by some authors in the literature (see, e.g., [2-6]); however, these works address the problem under a deterministic perspective, i.e., without considering the effects of uncertainties during the optimization procedure.

Similarly, handling manufacturing uncertainty in topology optimization is a challenging subject by itself. Manufacturing error tolerant topology optimization was first addressed in the literature in [7, 8], where a three-field robust approach was proposed to consider uniform boundary variations in topology optimization problems, including compliant mechanism design. Since then, several approaches have been proposed to handle manufacturing uncertainty in topology optimization (see, e.g., [9-16]); however, none of them addresses the stress-constrained topology design problem of compliant mechanisms.

Both manufacturing uncertainty and stress constraints in compliant mechanisms design have already been addressed separately in the literature. However, to the authors' best knowledge, there is no work which combines both approaches into a unified formulation, in order to achieve manufacturing tolerant compliant mechanisms that satisfy a stress failure criterion.

A unified approach, combining the stress-constrained design problem and the manufacturing tolerant three-field robust design approach has already been proposed in [17], where the volume minimization problem with stress constraints is addressed. However, volume minimization and compliant mechanism problems are essentially different from a design point of view, thereby motivating the investigation of such a unified formulation on the compliant mechanism design problem.

Uniform manufacturing uncertainty is a very relevant and physics-based uncertainty in microfabrication, where compliant mechanisms are manufactured by photo lithography and chemical etching processes. Over- or under-exposure and over- or under-etching are common sources of uncertainty in this field and motivated the robust formulation by [7]. Deeper investigations of these effects together with actual physical modeling of micro- and nano-lithography processes as a part of the topology optimization process have been discussed in [18-21]. Non-uniform manufacturing uncertainties have also been investigated for compliant mechanism design by [11], but the conclusion of that paper was that this did not result in significantly different designs compared to the uniform variations approach. Hence, here we limit ourselves to the computationally much more tractable uniform variations approach.

This work aims at proposing such a unified formulation, in order to address both manufacturing uncertainty and stress failure criterion on the design of compliant mechanisms through topology optimization. The proposed formulation is an extension of the three-field robust approach for compliant mechanism design presented in [8]. The novelty in this proposal comes from inclusion of a stress failure criterion in each projected field, following [17], in order to ensure compliant mechanisms that satisfy the stress failure criterion even in the presence of uniform boundary 
variations.

\section{Manufacturing tolerant compliant mechanism design}

In this work, the traditional density approach to topology optimization is employed [22]: the structural problem is solved with the displacement-based finite element method for linear elasticity under static loads [23]. Each finite element $e$ is associated with a physical relative density $\bar{\rho}_{e}$ varying from 0 (which represents void) to 1 (which represents solid). Once the structural optimization problem is defined (objective function and design constraints), a gradient-based algorithm is employed to find the set of relative densities (topology) which minimizes the objective function and satisfies the applied design constraints.

In this paper, the compliant mechanism design problem is studied. More specifically, we address the robust formulation presented by [8], which was proposed to handle uniform manufacturing uncertainties in topology optimization, including the compliant mechanism design problem.

Subsection 2.1 briefly describes the traditional robust formulation from [8] for topology design of compliant mechanisms. Subsection 2.2 describes the robust formulation proposed in this paper, which consists in an extension of the traditional robust formulation to deal with local stress constraints.

\subsection{Traditional robust approach}

The robust formulation proposed by [8] considers three sets of relative densities during the topology optimization procedure: $\overline{\boldsymbol{\rho}}^{(e)}, \overline{\boldsymbol{\rho}}^{(i)}$ and $\overline{\boldsymbol{\rho}}^{(d)}$; which represent eroded, intermediate and dilated topologies, respectively. The intermediate topology is the robust solution in the end of the optimization procedure, whereas both eroded and dilated topologies represent extreme manufacturing errors. The compliant mechanism design problem is formulated as a min/max problem [8]

$$
\begin{array}{ll}
\underset{\boldsymbol{\rho}}{\operatorname{Min} .} & \max \left(u_{\text {out }}\left(\overline{\boldsymbol{\rho}}^{(e)}\right), u_{\text {out }}\left(\overline{\boldsymbol{\rho}}^{(i)}\right), u_{\text {out }}\left(\overline{\boldsymbol{\rho}}^{(d)}\right)\right) \\
\text { s. t. } & V_{f}\left(\overline{\boldsymbol{\rho}}^{(d)}\right) \leqslant V_{u p}^{(d)} \\
& \mathbf{K}\left(\overline{\boldsymbol{\rho}}^{(e)}\right) \mathbf{U}\left(\overline{\boldsymbol{\rho}}^{(e)}\right)=\mathbf{F} \\
& \mathbf{K}\left(\overline{\boldsymbol{\rho}}^{(i)}\right) \mathbf{U}\left(\overline{\boldsymbol{\rho}}^{(i)}\right)=\mathbf{F} \\
& \mathbf{K}\left(\overline{\boldsymbol{\rho}}^{(d)}\right) \mathbf{U}\left(\overline{\boldsymbol{\rho}}^{(d)}\right)=\mathbf{F} \\
& 0 \leqslant \rho_{e} \leqslant 1
\end{array}
$$

where $\rho \in \mathbb{R}^{N_{e}}$ is the vector of design variables of the optimization problem, $N_{e}$ is the number of elements employed in the discretization of the design domain, $u_{\text {out }}\left(\overline{\boldsymbol{\rho}}^{(j)}\right)=\boldsymbol{\Lambda}^{T} \mathbf{U}\left(\overline{\boldsymbol{\rho}}^{(j)}\right)$ is the output displacement of the compliant mechanism 
for $j \in\{e, i, d\}$, which depends on the localization vector $\boldsymbol{\Lambda}$, which takes either 1 or -1 at the degree of freedom that corresponds to the output degree of freedom and zero otherwise, $V_{f}\left(\overline{\boldsymbol{\rho}}^{(d)}\right)=\frac{V\left(\overline{\boldsymbol{\rho}}^{(d)}\right)}{V_{\text {domain }}}$ is the volume fraction of the dilated design, $V\left(\overline{\boldsymbol{\rho}}^{(d)}\right)=\sum_{e=1}^{N_{e}} V_{e} \bar{\rho}_{e}^{(d)}$ is the structural volume of the dilated topology, $V_{e}$ is the volume of element $e, V_{d o m a i n}$ is the volume of the design domain, $V_{u p}^{(d)}$ is the upper volume fraction for the dilated design, $\mathbf{K}$ is the global stiffness matrix, $\mathbf{U}$ is the global displacement vector and $\mathbf{F}$ is the global load vector. The global stiffness matrix is assembled for each relative density field $\overline{\boldsymbol{\rho}}^{(j)}$, for $j \in\{e, i, d\}$, as $\mathbf{K}(\overline{\boldsymbol{\rho}})=\sum_{e=1}^{N_{e}} \mathbf{k}_{e}\left(\bar{\rho}_{e}\right)+k_{\text {in }}+k_{\text {out }}$, considering the stiffness matrix $\mathbf{k}_{e}$ of each element $e$, the input stiffness $k_{\text {in }}$, and the output stiffness $k_{\text {out }}$. The local stiffness matrix of each finite element $e$ is computed as $\mathbf{k}_{e}\left(\bar{\rho}_{e}\right)=\left(\rho_{\min }+\left(1-\rho_{\min }\right) \bar{\rho}_{e}^{p}\right) \mathbf{k}_{e}^{0}$, where $\rho_{\min }=1 \times 10^{-9}$ is adopted to avoid singularity of the global stiffness matrix when solving for equilibrium, $p$ is a penalization parameter (usually chosen equal to 3 in the literature, and also in this paper) related to the SIMP (Solid Isotropic Material with Penalization [24, 25]) interpolation, and $\mathbf{k}_{e}^{0}$ is the local stiffness matrix of element $e$ considering solid material.

Note that the volume constraint is applied over the dilated topology in this formulation, following [8]. As discussed in [7], when applying the volume constraint directly over the intermediate topology, some numerical instabilities may arise on the dilated design, leading to non-robust solutions. In this implementation we follow the suggestion proposed in [8], where the volume fraction is updated as $V_{u p}^{(d)}=\frac{V_{u p}^{(i)}}{V\left(\overline{\boldsymbol{\rho}}^{(i)}\right)} V\left(\overline{\boldsymbol{\rho}}^{(d)}\right)$ in every few iterations (see subsection 3 for details regarding this updating scheme), so the volume of the intermediate structure reaches the prescribed value in the end of the optimization procedure, assuming the volume constraint is active.

In this formulation, relative densities are related to design variables through density filtering with threshold projection [8]. The relative density of element $e, \bar{\rho}_{e}$, is computed as

$$
\bar{\rho}_{e}=\frac{\tanh (\beta \eta)+\tanh \left(\beta\left(\tilde{\rho}_{e}-\eta\right)\right)}{\tanh (\beta \eta)+\tanh (\beta(1-\eta))}
$$

where $\tilde{\rho}_{e}$ is the filtered relative density of element $e$, obtained from a linear projection

$$
\tilde{\rho}_{e}=\frac{\sum_{i \in \vartheta_{e}} w\left(\mathbf{x}_{i}\right) V_{i} \rho_{i}}{\sum_{i \in \vartheta_{e}} w\left(\mathbf{x}_{i}\right) V_{i}}
$$

over the design variables $\rho$, in a circular neighborhood $\vartheta_{e}$, centered in element $e$, which contains all the elements whose centers are within a radius $R$ specified by the designer.

The linear weighting function is defined as

$$
w\left(\mathbf{x}_{i}\right)=R-\left\|\mathbf{x}_{i}-\mathbf{x}_{e}\right\|
$$


where $\mathbf{x}_{i}$ are the coordinates of the center of element $i$ and $\mathbf{x}_{e}$ are the coordinates of the center of the neighborhood $\vartheta_{e}$.

The three sets of relative densities considered in this formulation, $\overline{\boldsymbol{\rho}}^{(e)}, \overline{\boldsymbol{\rho}}^{(i)}$ and $\overline{\boldsymbol{\rho}}^{(d)}$, represent the physical densities and actual topologies of the problem. These are obtained with Equation (2) for different values of $\eta$, considering the following relation: $\eta_{e}>\eta_{i}>\eta_{d}$. These three sets of relative densities are obtained from the same set of filtered densities, $\tilde{\boldsymbol{\rho}}$, which in turn are obtained from a single set of design variables, $\boldsymbol{\rho}$, by Equation (3).

The parameter $\beta>0$ in Equation (2) controls the nonlinearity of the threshold projection. For $\beta \rightarrow 0$ we have a linear behavior between relative and filtered densities, whereas for $\beta \rightarrow \infty$ we have a Heaviside step function [8]. Parameter $\beta$ is updated through a continuation strategy during the optimization procedure, as usually performed in projection-based formulations to topology optimization $[8,25,26]$, starting from a small value $\beta^{(1)}$ up to a maximum value $\beta_{\max }$ defined by the designer. Parameter $\beta_{\max }$ is usually chosen large enough to ensure crisp black and white solutions [8]. In this paper we follow the hints proposed in [17] to define the value of $\beta_{\max }$, by choosing it in order to allow a thin smooth transition boundary of intermediate material between solid and void phases. In [17], it is demonstrated that a thin smooth transition of intermediate material, associated with a proper stress interpolation function, ensures stress accuracy and low stress oscillation after uniform boundary variations. Although the traditional formulation described in this subsection (from [8]) does not take stress constraints into account, we choose to use the same parameters for computing the projection functions in order to ensure a fair comparison between both traditional and proposed formulations. Da Silva et al. [17] propose a parameter $\beta_{\text {lim }}=2 R / l_{e}$, where $l_{e}$ is the length of the side of a square element considering a fixed grid discretization of the design domain, in order to achieve a smooth transition with width equal to at least the size of one finite element. The authors [17] also show that a slightly thicker transition layer, ensured by defining $\beta_{\max }=\beta_{\text {lim }} / 2$, associated with a reasonable choice for interpolating the stresses, is sufficient to ensure stress accuracy on the boundaries of the topology; thus, in this paper we employ this same $\beta_{\max }$ parameter for solving all the numerical examples (with or without stress constraints).

The parameter $\eta \in[0,1]$, in Equation (2), controls the inflexion point of the projection, defining the amount of filtered densities $\tilde{\rho}_{e}$ projected to either 0 or 1 , for $\beta \rightarrow \infty$. For $\eta=0$, for instance, we recover the Heaviside step approximation proposed in [26]. For $\eta=1$, we recover the modified Heaviside step approximation proposed in [25].

In the traditional formulation, Equation (1), the worst of the three output displacements is optimized. The objective function takes both eroded and dilated projections into consideration, besides the intermediate projection, during the optimization procedure; thus avoiding these same projections to result in possible manufacturing errors, i.e., avoiding a disconnected eroded topology and a too stiff dilated topology [8]. 


\subsection{Proposed robust approach with stress constraints}

This subsection proposes an extension to the traditional robust formulation, Equation (1), in order to include a stress failure criterion. Based on the robust approach proposed in [17], where the stress constraints are simultaneously applied over eroded, intermediate and dilated designs, we propose the following stress-robust optimization problem for compliant mechanism design considering the von Mises failure criterion

$$
\begin{aligned}
& \underset{\boldsymbol{\rho}}{\operatorname{Min} .} \quad \frac{k_{\text {in }}}{f_{\text {in }}} \max \left(u_{\text {out }}\left(\overline{\boldsymbol{\rho}}^{(e)}\right), u_{\text {out }}\left(\overline{\boldsymbol{\rho}}^{(i)}\right), u_{\text {out }}\left(\overline{\boldsymbol{\rho}}^{(d)}\right)\right)+k_{v} V_{f}\left(\overline{\boldsymbol{\rho}}^{(d)}\right) \\
& \text { s. t. } \quad V_{f}\left(\overline{\boldsymbol{\rho}}^{(d)}\right) \leqslant V_{u p}^{(d)} \\
& V_{f}\left(\overline{\boldsymbol{\rho}}^{(d)}\right) \geqslant V_{\text {low }}^{(d)} \\
& \frac{\sigma_{e q}^{(k)}\left(\overline{\boldsymbol{\rho}}^{(e)}\right)}{\sigma_{y}}-1 \leqslant 0 \\
& \frac{\sigma_{e q}^{(k)}\left(\overline{\boldsymbol{\rho}}^{(i)}\right)}{\sigma_{y}}-1 \leqslant 0 \\
& \frac{\sigma_{e q}^{(k)}\left(\overline{\boldsymbol{\rho}}^{(d)}\right)}{\sigma_{y}}-1 \leqslant 0 \\
& k=1,2, \ldots, N_{k} \\
& \mathbf{K}\left(\overline{\boldsymbol{\rho}}^{(e)}\right) \mathbf{U}\left(\overline{\boldsymbol{\rho}}^{(e)}\right)=\mathbf{F} \\
& \mathbf{K}\left(\overline{\boldsymbol{\rho}}^{(i)}\right) \mathbf{U}\left(\overline{\boldsymbol{\rho}}^{(i)}\right)=\mathbf{F} \\
& \mathbf{K}\left(\overline{\boldsymbol{\rho}}^{(d)}\right) \mathbf{U}\left(\overline{\boldsymbol{\rho}}^{(d)}\right)=\mathbf{F} \\
& 0 \leqslant \rho_{e} \leqslant 1 \\
& k=1,2, \ldots, N_{k} \text {, } \\
& k=1,2, \ldots, N_{k} \\
& e=1,2, \ldots, N_{e}
\end{aligned}
$$

where $f_{\text {in }} / k_{\text {in }}$ is the free displacement of the input actuator, which depends on both the input load $f_{\text {in }}$ and input stiffness $k_{i n}$, employed here for the purpose of normalization, $k_{v}$ is a weighting parameter defined by the designer, $V_{l o w}^{(d)}$ is the lower volume fraction for the dilated design, $\sigma_{e q}^{(k)}\left(\overline{\boldsymbol{\rho}}^{(j)}\right)$ is the von Mises equivalent stress computed at point $k$, for $j \in\{e, i, d\}, \sigma_{y}$ is the yield stress, and $N_{k}$ is the number of points of stress computation.

The von Mises equivalent stress at any point $k$ is computed based on [1], and is written as

$$
\sigma_{e q}^{(k)}(\overline{\boldsymbol{\rho}})=f_{\sigma}\left(\bar{\rho}_{k}\right) \sqrt{\hat{\boldsymbol{\sigma}}_{k}^{T}(\overline{\boldsymbol{\rho}}) \mathbf{M} \hat{\boldsymbol{\sigma}}_{k}(\overline{\boldsymbol{\rho}})+\sigma_{\min }^{2}}
$$

where $f_{\sigma}\left(\bar{\rho}_{k}\right)$ is the stress interpolation function, $\hat{\boldsymbol{\sigma}}_{k}(\overline{\boldsymbol{\rho}})$ is the solid stress vector at point $k, \sigma_{\min }=1 \times 10^{-4} \sigma_{y}$ is a small value included in our implementations to ensure a positive von Mises equivalent stress when $\hat{\boldsymbol{\sigma}}_{k}^{T}(\overline{\boldsymbol{\rho}}) \mathbf{M} \hat{\boldsymbol{\sigma}}_{k}(\overline{\boldsymbol{\rho}}) \rightarrow 0$, thus avoiding numerical instabilities during the sensitivity analysis, needed for optimization with gradient-based algorithm, 
and $\mathbf{M}$ is a constant matrix, defined for plane stress problems as

$$
\mathbf{M}=\left[\begin{array}{ccc}
1 & -\frac{1}{2} & 0 \\
-\frac{1}{2} & 1 & 0 \\
0 & 0 & 3
\end{array}\right]
$$

In this paper, we employ the $\varepsilon$-relaxed approach [27] to relax the stress constraints, thus avoiding the singularity phenomenon, adapted to solve density-based problems by [28], with $f_{\sigma}\left(\bar{\rho}_{k}\right)=\frac{\bar{\rho}_{k}}{\varepsilon\left(1-\bar{\rho}_{k}\right)+\bar{\rho}_{k}}$. Da Silva et al. [17] demonstrated, through numerical investigation, that the choice of $\varepsilon$, when associated with a of smooth transition boundary of proper thickness between solid and void phases, can ensure stress accuracy and low stress oscillation after uniform boundary variation. In general, it was observed that when $\varepsilon$ is too small, the stresses at the jagged edges of the structures are overestimated and strong stress oscillations may occur, whereas that when $\varepsilon$ is too large, the stresses are underestimated. Following [17], we choose $\varepsilon=0.2$, in order to improve accuracy of stress evaluation at topology boundaries.

The solid stress vector at point $k$ is computed as

$$
\hat{\boldsymbol{\sigma}}_{k}(\overline{\boldsymbol{\rho}})=\mathbf{C}^{0} \mathbf{B}_{k} \mathbf{u}_{k}(\overline{\boldsymbol{\rho}})
$$

where $\mathbf{C}^{0}$ is the constitutive matrix of base material, $\mathbf{B}_{k}$ is the strain-displacement transformation matrix evaluated at point $k$ and $\mathbf{u}_{k}(\bar{\rho})$ is the local displacement vector of the element which contains point $k$.

The differences between traditional and proposed approaches, Equations (1) and (5), respectively, are described next:

1. The stress constraints. The greatest difference between both approaches are the stress constraints, that are not considered in the traditional formulation. In the proposed formulation, the structural design associated with each projected field is subjected to local stress constraints, which may greatly increase the size of the optimization problem. For instance, considering a mesh with 10k elements, and one point of stress computation per element, we would have an optimization problem with 10k design variables and 30k stress constraints, besides the volume and bound constraints.

2. The weighting factor $k_{v}$. As noted in [2], when solving the deterministic stress-constrained compliant mechanism design problem, if very strong requirements to stress are imposed (i.e., a too small $\sigma_{y}$ ), the optimized structure will be very slender, and hence the (upper) volume constraint is not used. In this paper, we experienced the same behavior when solving the robust version of the same problem, when considering small stress 
limits. However, in the robust case, when the upper volume constraint is not active, numerical instabilities may arise. This phenomenon was first observed in [7] when trying to apply the volume constraint directly over the intermediate topology. Sigmund [7] demonstrated that, when the volume of the dilated topology is not constrained, there is freedom for the algorithm to place superfluous material over the optimized topology, leading to non-optimal designs. In this paper, we follow the hints proposed in [8], in the sense that the upper volume constraint is applied over the dilated design and is updated every few iterations, as described after Equation (1). However, when stress constraints are considered in the robust compliant mechanism problem, even though the dilated volume is constrained, sometimes this constraint is not active in the final design, due to the strong stress requirements, leading, again, to superfluous material placement. In order to alleviate this issue, we included the volume fraction of the dilated topology in the objective function, weighted by a constant $k_{v}$ defined by the designer. As shown later in subsection 4.1, the choice of $k_{v}$ plays an important role in problem solution, and it should be properly chosen in order to avoid placement of superfluous material over the dilated design, and to ensure good performance (regarding the output displacement) of the mechanism.

3. The lower volume fraction $V_{\text {low }}^{(d)}$. When the dilated volume is included as objective, the optimizer sometimes prefers to minimize the volume, rather than the output displacements, at the first steps of the optimization procedure, getting stuck in a void design (relative densities equal to zero). Since the design is feasible for the void situation (zero density means zero stresses, due to the stress interpolation), the optimizer sees the trivial solution as a local minimum point. The lower volume fraction acts as a numerical means, preventing this void situation, by enforcing a minimum amount of solid material in the solution. The lower volume constraint, however, is important only in the first few iterations, while the topology is forming. Moreover, in the problems analyzed in this paper, the lower volume constraint is not active in the final designs, indicating that this constraint acts only in avoiding trivial designs, and has no influence on the performance of the optimized mechanisms.

4. The weighting factor $k_{i n} / f_{i n}$. The output displacements are weighted by the inverse of the free displacement of the input actuator for the purpose of normalization. This normalization aims at reducing the dependence of the parameter $k_{v}$ on the problem.

\section{Solution algorithm}

It is acknowledged, in the literature, that stress-constrained continuum topology optimization problems are very challenging, mainly due to the local nature of the stress failure criterion, which leads to large optimization problems, with a large number of design variables and a large number of stress constraints. Some techniques were proposed over the years to handle the large number of stress constraints resulting from such formulations: aggregation techniques 
[28-30], active set strategies [1, 31], and the augmented Lagrangian method [32, 33]; to name a few. As discussed in [17], the major difference between deterministic and robust stress-constrained formulations, from a computational point of view, is in the larger number of stress constraints in the robust case (three times larger). Thus, the optimization problems resulting from the proposed formulation, Equation (5), may be addressed by any of these aforementioned approaches as well.

The numerical examples addressed in this paper are solved with the augmented Lagrangian method described in [34]. The augmented Lagrangian method has been employed, in the literature (see, e.g., [17, 32, 33, 35-42]), as an alternative approach to the commonly used stress constraint aggregation techniques.

The augmented Lagrangian method is given by a sequential solution of several optimization subproblems. The objective function of the optimization subproblems is the augmented Lagrangian function, which is defined as a weighted sum between the objective function of the original problem (output displacements and the volume fraction of the dilated design) and applied design constraints (stress and volume constraints) weighted by penalization parameters and associated with Lagrange multipliers. After solving a given optimization subproblem, Lagrange multipliers and penalization parameters are updated, and then, the next subproblem is solved, and so on, until the convergence criteria are reached.

Before presenting the augmented Lagrangian function for the robust approach, we propose the use of the Kreisselmeier-Steinhauser (KS) function [43] to smooth the maximum operator applied over the output displacements of eroded, intermediate and dilated topology, as

$$
u_{\text {out }}^{K S}\left(\overline{\boldsymbol{\rho}}^{(e)}, \overline{\boldsymbol{\rho}}^{(i)}, \overline{\boldsymbol{\rho}}^{(d)}\right)=\frac{1}{P} \ln \left(\sum_{j \in\{e, i, d\}} \exp \left(P u_{\text {out }}\left(\overline{\boldsymbol{\rho}}^{(j)}\right)\right)\right),
$$

with $u_{\text {out }}^{K S}\left(\overline{\boldsymbol{\rho}}^{(e)}, \overline{\boldsymbol{\rho}}^{(i)}, \overline{\boldsymbol{\rho}}^{(d)}\right) \rightarrow \max \left(u_{\text {out }}\left(\overline{\boldsymbol{\rho}}^{(e)}\right), u_{\text {out }}\left(\overline{\boldsymbol{\rho}}^{(i)}\right), u_{\text {out }}\left(\overline{\boldsymbol{\rho}}^{(d)}\right)\right)$ for $P \rightarrow \infty$. In practical implementations, $P>0$ should be large enough to ensure a good approximation for the maximum operator. This smooth approximation is employed to allow continuous differentiability of the objective function; also allowing solution of the optimization subproblems by a gradient-based algorithm. An alternative would be use of the bound formulation presented in [44], as usually employed in the literature when solving the traditional robust problem [7, 8]. In the numerical examples addressed in this paper, the KS function with $P=10$ is employed, since it provides a good compromise between accuracy and smoothness.

Considering the smooth approximation for the maximum output displacement, Equation (9), we defined the 
augmented Lagrangian function associated with the proposed robust formulation, Equation (5), as

$$
\begin{gathered}
L\left(\overline{\boldsymbol{\rho}}^{(e)}, \overline{\boldsymbol{\rho}}^{(i)}, \overline{\boldsymbol{\rho}}^{(d)}, \boldsymbol{\mu}, \mathbf{r}\right)=\frac{k_{\text {in }}}{f_{\text {in }}} u_{\text {out }}^{K S}\left(\overline{\boldsymbol{\rho}}^{(e)}, \overline{\boldsymbol{\rho}}^{(i)}, \overline{\boldsymbol{\rho}}^{(d)}\right)+k_{v} V_{f}\left(\overline{\boldsymbol{\rho}}^{(d)}\right)+\frac{r_{u p}}{2}\left\langle\frac{\mu_{u p}}{r_{u p}}+\frac{V_{f}\left(\overline{\boldsymbol{\rho}}^{(d)}\right)}{V_{u p}^{(d)}}-1\right\rangle^{2} \\
+\frac{r_{\text {low }}}{2}\left\langle\frac{\mu_{\text {low }}}{r_{\text {low }}}-\frac{V_{f}\left(\overline{\boldsymbol{\rho}}^{(d)}\right)}{V_{\text {low }}^{(d)}}+1\right\rangle^{2}+\frac{r}{2} \sum_{k=1}^{N_{k}}\left(\left\langle\frac{\mu_{k}^{(d)}}{r}+\frac{\sigma_{e q}^{(k)}\left(\overline{\boldsymbol{\rho}}^{(d)}\right)}{\sigma_{y}}-1\right\rangle^{2}\right. \\
\left.\left.+\left\langle\frac{\mu_{k}^{(i)}}{r}+\frac{\sigma_{e q}^{(k)}\left(\overline{\boldsymbol{\rho}}^{(i)}\right)}{\sigma_{y}}-1\right\rangle^{2}+\left\langle\frac{\mu_{k}^{(e)}}{r}+\frac{\sigma_{e q}^{(k)}\left(\overline{\boldsymbol{\rho}}^{(e)}\right)}{\sigma_{y}}-1\right\rangle\right\rangle^{2}\right)
\end{gathered}
$$

where $\langle\cdot\rangle=\max (0, \cdot), \boldsymbol{\mu}$ is a vector which contains all Lagrange multipliers of the problem: $\mu_{u p}$, associated with the upper volume constraint; $\mu_{\text {low }}$, associated with the lower volume constraint; and $\mu_{k}^{(j)}$, for $j \in\{e, i, d\}$ and $k=1,2, \ldots, N_{k}$, associated with the stress constraints; and $\mathbf{r}$ is a vector which contains the penalization parameters: $r_{u p}$, associated with the upper volume constraint; $r_{l o w}$, associated with the lower volume constraint; and $r$, associated with all the stress constraints.

The optimization subproblems are then formulated as

$$
\begin{array}{cll}
\underset{\boldsymbol{\rho}}{\operatorname{Min}} & L\left(\overline{\boldsymbol{\rho}}^{(e)}, \overline{\boldsymbol{\rho}}^{(i)}, \overline{\boldsymbol{\rho}}^{(d)}, \boldsymbol{\mu}^{(c)}, \mathbf{r}^{(c)}\right) \\
& & \\
\text { s. t. } & \mathbf{K}\left(\overline{\boldsymbol{\rho}}^{(e)}\right) \mathbf{U}\left(\overline{\boldsymbol{\rho}}^{(e)}\right)=\mathbf{F} & \\
& \mathbf{K}\left(\overline{\boldsymbol{\rho}}^{(i)}\right) \mathbf{U}\left(\overline{\boldsymbol{\rho}}^{(i)}\right)=\mathbf{F} \\
& \mathbf{K}\left(\overline{\boldsymbol{\rho}}^{(d)}\right) \mathbf{U}\left(\overline{\boldsymbol{\rho}}^{(d)}\right)=\mathbf{F} & \\
& 0 \leqslant \rho_{e} \leqslant 1 \quad \quad e=1,2, \ldots, N_{e}
\end{array}
$$

where the superscript ${ }^{(c)}$ indicates $c$-th optimization subproblem.

After solving $c$-th optimization subproblem, the solution $\left(\boldsymbol{\rho}^{(c)}\right)^{*}$ of the current subproblem is employed to update the next estimate of Lagrange multipliers associated with volume and stress constraints, as

$$
\begin{aligned}
& \mu_{\text {up }}^{(c+1)} \leftarrow\left\langle r_{u p}^{(c)}\left(\frac{V_{f}\left(\left(\overline{\boldsymbol{\rho}}_{(c)}^{(d)}\right)^{*}\right)}{V_{u p}^{(d)}}-1\right)+\mu_{\text {low }}^{(c)}\right\rangle, \\
& \mu_{\text {low }}^{(c+1)} \leftarrow\left\langle r_{\text {low }}^{(c)}\left(-\frac{V_{f}\left(\left(\overline{\boldsymbol{\rho}}_{(c)}^{(d)}\right)^{*}\right)}{V_{\text {low }}^{(d)}}+1\right)+\mu_{\text {low }}^{(c)}\right\rangle,
\end{aligned}
$$


and

$$
\left(\mu_{k}^{(j)}\right)^{(c+1)} \leftarrow\left\langle r^{(c)}\left(\frac{\sigma_{e q}^{(k)}\left(\left(\overline{\boldsymbol{\rho}}_{(c)}^{(j)}\right)^{*}\right)}{\sigma_{y}}-1\right)+\left(\mu_{k}^{(j)}\right)^{(c)}\right\rangle,
$$

for $j \in\{e, i, d\}$, and penalization parameter associated with the stress constraints only, as

$$
r^{(c+1)} \leftarrow \begin{cases}\min \left(\gamma r^{(c)}, r_{\max }\right) & \text { if } \delta \sigma_{\max }^{(c)}>\omega \delta \sigma_{\max }^{(c-1)} \\ r^{(c)} & \text { otherwise }\end{cases}
$$

where $\gamma>1$ and $\omega<1$ are update parameters, $r_{\max }$ is an upper value for the penalization parameter and $\delta \sigma_{\max }=$ $\left(\frac{\sigma_{\max }}{\sigma_{y}}-1\right)$, where $\sigma_{\max }$ is the maximum value among all computed von Mises equivalent stresses. Penalization parameters associated with upper and lower volume constraints, $r_{u p}$ and $r_{l o w}$, respectively, are kept constant throughout the optimization procedure, since we experienced no need to increase these values in order to achieve feasibility regarding the structural volume.

The augmented Lagrangian method is a continuation approach itself, in the sense that it consists of solving the original problem with a sequence of modified subproblems. This continuation approach leads to a sequential updating strategy regarding the Lagrange multipliers and penalization parameter, since these parameters are updated every time an optimization subproblem is solved. As discussed in subsection 2.1, a similar sequential strategy is often employed in projection-based approaches to topology optimization, where parameter $\beta$, which controls the nonlinearity of the threshold projection, is usually updated through a continuation approach, from a small value to a maximum one. Thus, we employ two distinct continuation strategies in this paper: 1) for updating the Lagrange multipliers $\boldsymbol{\mu}$, Equations (12), (13) and (14), penalization parameter $r$, Equation (15), and volume fraction of dilated design, as $V_{u p}^{(d)}=\frac{V_{u p}^{(i)}}{V\left(\overline{\boldsymbol{\rho}}^{(i)}\right)} V\left(\overline{\boldsymbol{\rho}}^{(d)}\right)$; and 2 ) for updating parameter $\beta$, as $\beta=\min \left(2 \beta, \beta_{\max }\right)$. Updating criteria are shown next:

- if $\left(\|\Delta \rho\|_{\infty}^{s u b}<0.01\right)$ or $\left(n_{i t}^{\text {sub }}=20\right)$, update $\boldsymbol{\mu}, r$ and $V_{u p}^{(d)}$;

- if $\left[\left(\|\Delta \rho\|_{\infty}^{\text {out }}<0.2\right)\right.$ and $\left.\left(\delta g_{\max }<0.01\right)\right]$ or $\left(n_{i t}^{\beta}=100\right)$, update $\beta$;

where $\|\Delta \rho\|_{\infty}^{s u b}$ is the maximum difference between to consecutive iterations of a given subproblem, $n_{i t}^{s u b}$ is the number of iterations of a given subproblem, $\|\Delta \rho\|_{\infty}^{\text {out }}$ is the maximum difference between solutions of two consecutive subproblems, $\delta g_{\max }=\max \left(\frac{V_{f}}{V_{u p}}-1,-\frac{V_{f}}{V_{\text {low }}}+1, \frac{\sigma_{\max }}{\sigma_{y}}-1\right)$ is the maximum constraint violation, and $n_{i t}^{\beta}$ is the number of iterations for a given value of $\beta$.

The stopping criteria are applied when $\beta=\beta_{\max }$, and are as follows:

- if $\left(n_{i t}^{\beta} \leqslant 100\right)$, stop the procedure if $\left(\|\Delta \rho\|_{\infty}^{o u t}<0.2\right)$ and $\left(\delta g_{\max }<0.01\right)$;

- if $\left(n_{i t}^{\beta}>100\right)$, stop the procedure if $\left(\delta g_{\max }<0.01\right)$. 
The augmented Lagrangian function is continuous and has continuous first-order derivatives [34, 45]; however, it has discontinuous second-order derivatives, since the maximum operator $\langle\cdot\rangle$ is non-differentiable at zero. Thus, the optimization subproblems, Equation (11), may be solved with any first-order algorithm able to handle bound constraints. In this paper, a modified steepest descent method, as described in [39], is employed, due to its simplicity, with minimum and maximum ranges of move limits as $\rho_{e} \pm 0.001$ and $\rho_{e} \pm 0.1$, respectively. Since the employed approach is gradient-based, sensitivity analysis of the augmented Lagrangian function with respect to the design variables is required. Sensitivity analysis is shown in the appendix.

\section{Numerical results}

In this section, several numerical experiments are performed over two test problems [46]: a) the inverter problem; and b) the gripper problem; Figure 1. Input data are taken from [8], with minor modifications to properly address the stress constraints. Input data defined next are employed throughout the results section, unless specified otherwise.

(a)

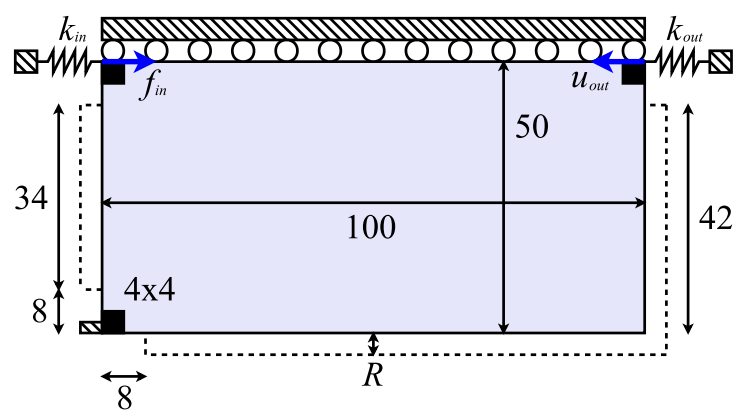

(b)

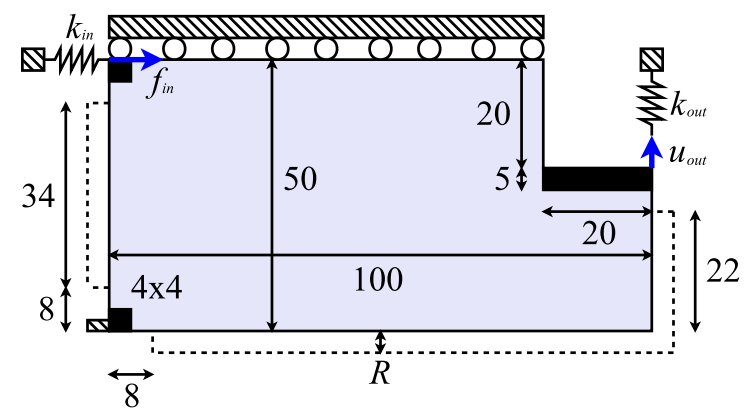

Figure 1: Inverter (a) and Gripper (b) design problems. Support regions on the bottom left of both design domains have size equal to 1 . Square-shaped fixed solid regions have size equal to $4 \times 4$.

Mechanic and geometric properties: Young's Modulus of 1, Poisson's ratio of 0.3, thickness of 1, applied load of $f_{i n}=1$, and input stiffness of $k_{\text {in }}=1$. The output stiffness is considered as: a) $k_{\text {out }}=0.001$, for the inverter problem; and b) $k_{\text {out }}=0.005$, for the gripper problem.

Design constraints: $V_{u p}=0.3$ and $V_{l o w}=0.2$ as upper and lower volume fractions, respectively. The addressed problems are solved for four distinct situations regarding the stress constraints: no stress constraints; $\sigma_{y}=0.01$; $\sigma_{y}=0.008 ;$ and $\sigma_{y}=0.006$.

Optimization parameters and mesh information: $r_{u p}=1, r_{\text {low }}=10, r^{(1)}=1 / N_{e}, r_{\max }=1 \times 10^{5} / N_{e}, \gamma=2$ and $\omega=0.8$, associated with the augmented Lagrangian method; initial value of design variables of $\boldsymbol{\rho}^{(1)}=V_{u p} \mathbf{1}$; filter's radius of $R=2.8$; and finite element meshes with $N_{e}=80,000$ (inverter) and $N_{e}=73,600$ (gripper) four-node bi-linear 
square elements (i.e., $400 \times 200$ elements). For this filter's radius and these finite element discretizations, parameter $\beta^{(1)}=1$ is updated up to a maximum value $\beta_{\max }=\beta_{\text {lim }} / 2=11.2$. Parameters $\eta_{d}, \eta_{i}$ and $\eta_{e}$ are chosen as: $0.25,0.5$ and 0.75 (inverter); and 0.3, 0.5 and 0.7 (gripper). The von Mises equivalent stresses are evaluated at the centroid of each finite element. The filter boundary padding scheme [47] is employed in order to alleviate possible boundary effects that may occur due to filtering, by extending the design domain with void elements (dashed regions in Figure 1), which are not considered in the analyses, only during application of the filter.

Both design domains are filled with fixed solid areas in load and support regions, as indicated in Figure 1. The stresses at these regions are set to zero, and thus not taken into account by the algorithm during the optimization procedure.

After solving the compliant mechanism design problems, the optimized topologies are post-processed through two different schemes:

1. Pixel-based. The projection function, Equation (2), is applied over the optimized filtered density fields for $\eta \in\left[\eta_{d}, \eta_{e}\right]$, considering incremental steps of 0.001 . The stresses are evaluated at the centroid of each element, and the maximum von Mises equivalent stress is then computed for each value of $\eta$ and compared with the yield stress $\sigma_{y}$.

2. Body-fitted. The contour plots extracted from the optimized filtered density fields associated with values of $\eta$ within the interval $\left[\eta_{d}, \eta_{e}\right]$, considering incremental steps of 0.05 , are employed to construct fine body-fitted finite element models with software Gmsh [48] comprised of six-node triangle elements. The von Mises equivalent stresses are computed at the centroid of each element, and the maximum stress for each $\eta$ is then compared with the yield stress $\sigma_{y}$.

The employment of two post-processing schemes aims at verifying if the pixel-based and body-fitted models have good agreement regarding maximum von Mises stress values, in range $\eta \in\left[\eta_{d}, \eta_{e}\right]$, and if the obtained intermediate designs are truly robust with respect to uniform boundary variations.

Topologies are illustrated in gray scale, Figure 2, where black represents solid $(\bar{\rho}=1)$ and white represents void $(\bar{\rho}=0)$. Von Mises stresses are illustrated in color images, Figure 3, where red represents maximum von Mises stress (which depends on the problem) and blue represents minimum von Mises stress $(=0)$.

Figure 2: Gray scale employed to represent topologies. White represents the void phase $(\bar{\rho}=0)$ and black the solid phase $(\bar{\rho}=1)$. 
Figure 3: Color scale employed to represent von Mises stresses. Red represents maximum (which depends on the problem) and blue minimum von Mises stress (=0).

\subsection{On the influence of parameter $k_{v}$}

This subsection aims at showing the influence of parameter $k_{v}$ over the optimized topologies. As discussed in subsection 2.1, if we formulate the traditional robust approach (Equation (1)) with the volume constraint applied directly over the intermediate design, superfluous material appears on the topologies leading to non-optimal solutions. A simple and effective approach that is often employed to avoid this issue is the enforcement of the volume constraint over the dilated structure, as suggested by [8] and also applied in this paper, taking care to update the volume of the dilated structure every few iterations, so the volume of the intermediate structure reaches the prescribed value at the end of the optimization procedure.

However, even though we apply the volume constraint over the dilated design, we may still have some issues when stress constraints are considered in the formulation. As discussed in subsection 2.2, when the stress-constrained compliant mechanism design problem is solved for very small stress limits, the volume constraint may not be active at the end of the optimization procedure. As shown in [2], the volume constraint not being active in the deterministic formulation is not an issue; however, it becomes an issue when addressing the stress-constrained problem through the three-field robust approach, as in this paper. It can be clearly seen in Figure 4, where the inverter problem is solved for $\sigma_{y}=0.006$ (the smallest yield stress value considered in this paper) and $k_{v}=0$ (i.e., the objective function is purely composed by the output displacements).

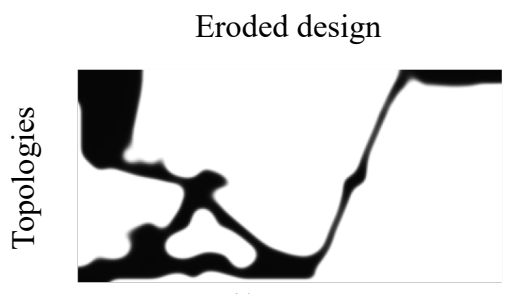

$V_{f}^{(e)}=0.1914$

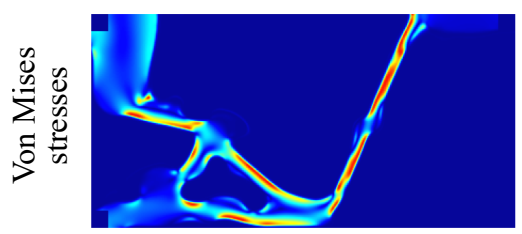

$\sigma_{\max }^{(e)}=0.00601$
Intermediate design

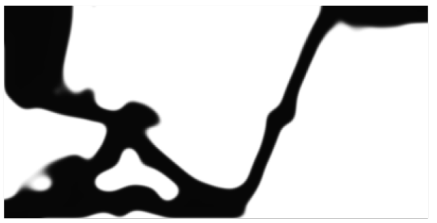

$V_{f}^{(i)}=0.2670$

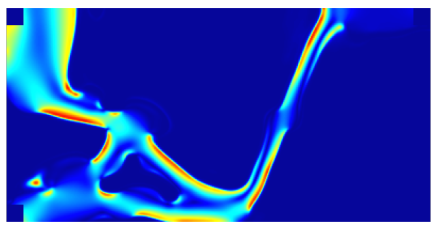

$\sigma_{\text {max }}^{(i)}=0.00600$
Dilated design

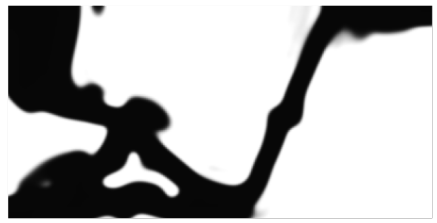

$V_{f}^{(d)}=0.3401$

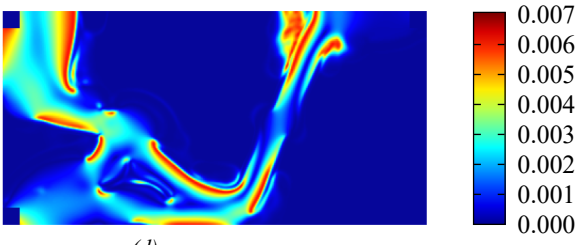

$\sigma_{\text {max }}^{(d)}=0.00604$

Figure 4: Inverter problem. Optimized designs for $\sigma_{y}=0.006$ and without penalizing the dilated volume, i.e., $k_{v}=0$.

In this case, the value of $\sigma_{y}$ is small, and hence, the volume of the intermediate topology is smaller than the volume 
fraction constraint of $V_{u p}=0.3$ at the end of the optimization procedure. Parameter $k_{v}$ thus plays an important role in the proposed formulation, Equation (5), which is clearly observed when solving the same problem considering several different values for $k_{v}$, Figure 5 .

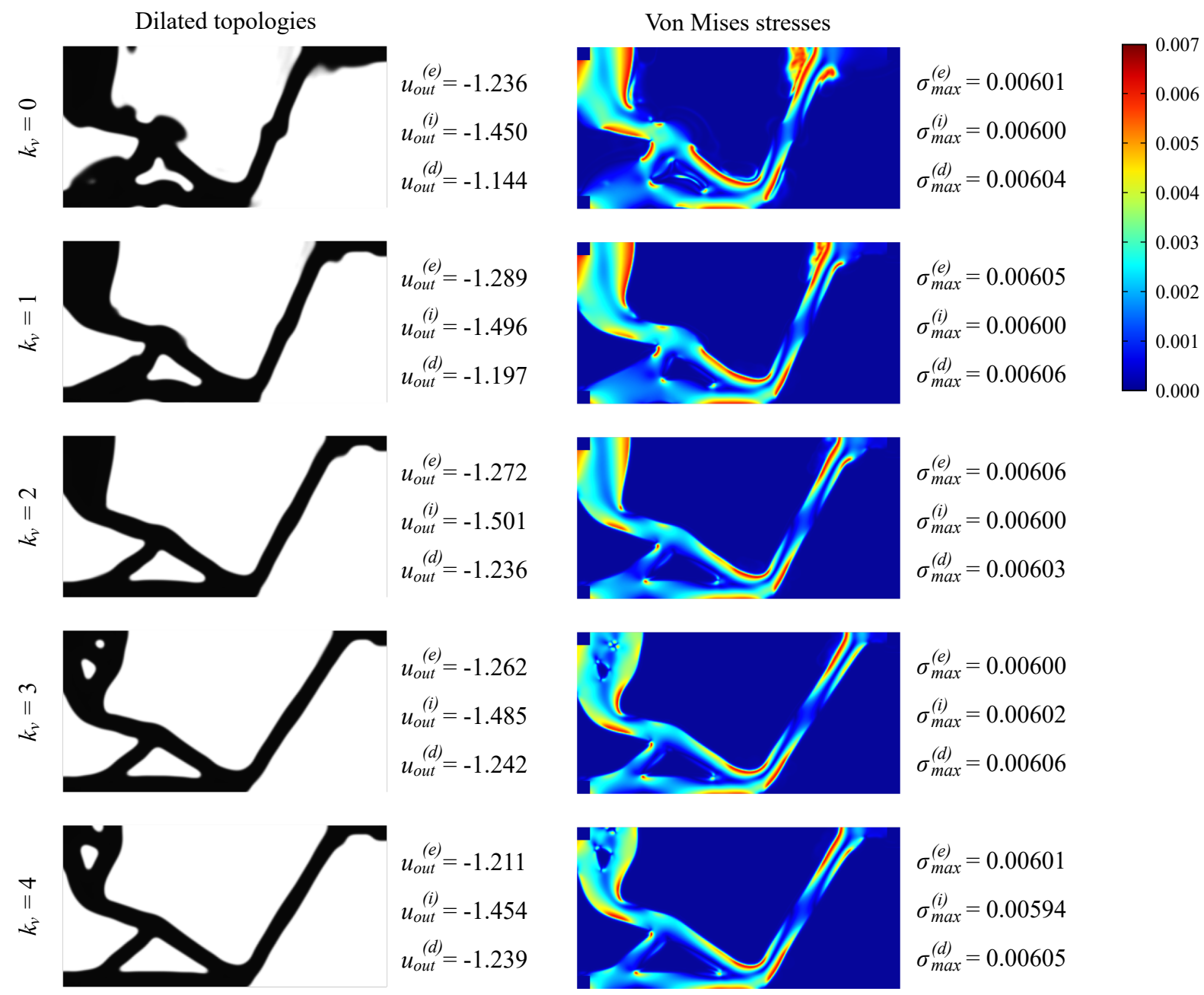

Figure 5: Inverter problem. Dilated designs for $\sigma_{y}=0.006$ and $k_{v}=0,1,2,3$ and 4.

Figure 5 shows the optimized dilated topologies with respective von Mises equivalent stresses. It is shown that the larger the value of $k_{v}$, the larger the cleaning effect over both dilated topology and respective von Mises stress field. This is justified, in this case, since parameter $k_{v}$ controls the amount of volume of the dilated design in the objective function; in this case, parameter $k_{v}$ acts at minimizing the volume of the dilated topology, thus avoiding this undesired effect of placement of superfluous material when the volume constraint is not active. However, one can see that if the value of $k_{v}$ is too large, the volume starts getting prioritized over the output displacements, which may affect performance of the optimized compliant mechanism. In this paper, we choose to work with $k_{v}=2$, since this value seems to provide a 
good compromise between performance (output displacements) and topology cleaning, in this example.

\subsection{Optimization results and post-processing}

In this subsection, both inverter and gripper problems, Figure 1, are addressed considering $k_{v}=2$ and four situations regarding the stress constraints: no stress constraints; $\sigma_{y}=0.01 ; \sigma_{y}=0.008$; and $\sigma_{y}=0.006$, respectively. Obtained results are then post-processed with both pixel-based and body-fitted approaches as described earlier.

\subsubsection{Inverter problem}

Figures 6 and 7 show optimized topologies (eroded, intermediate and dilated) and respective pixel-based von Mises equivalent stresses, for the inverter mechanism problem.

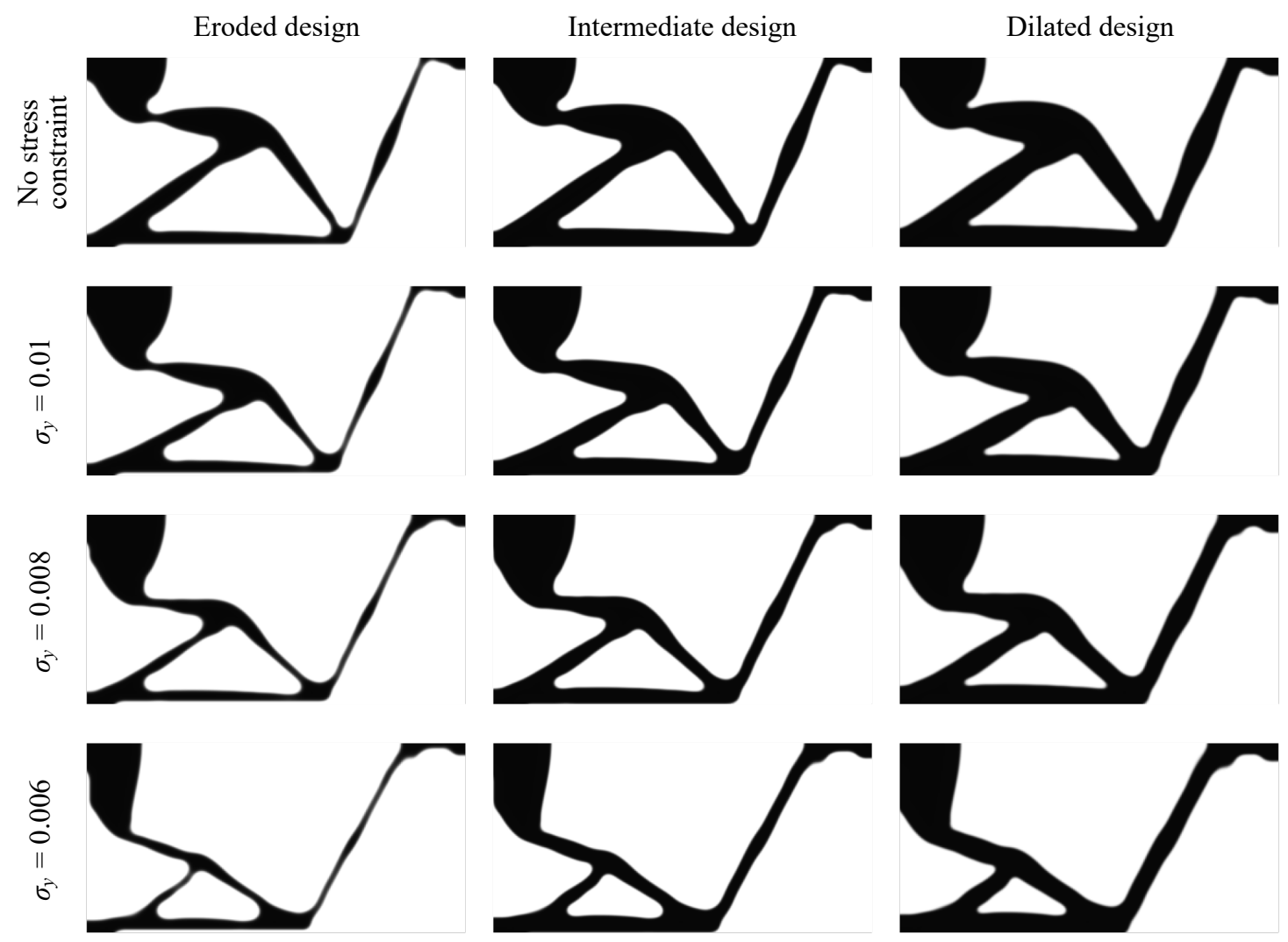

Figure 6: Inverter problem. Optimized topologies for $k_{v}=2$ and four levels of stress constraints: no stress constraints; $\sigma_{y}=0.01 ; \sigma_{y}=0.008 ;$ and $\sigma_{y}=0.006$.

By analyzing Figure 6, one can verify that although optimized topologies are the same, different shapes are obtained. The smaller the value of $\sigma_{y}$, the more rounded becomes the link between the structural member that connects the output and the body of the inverter. Figure 7 shows that, the smaller the value of $\sigma_{y}$, the more the stresses for the eroded, 

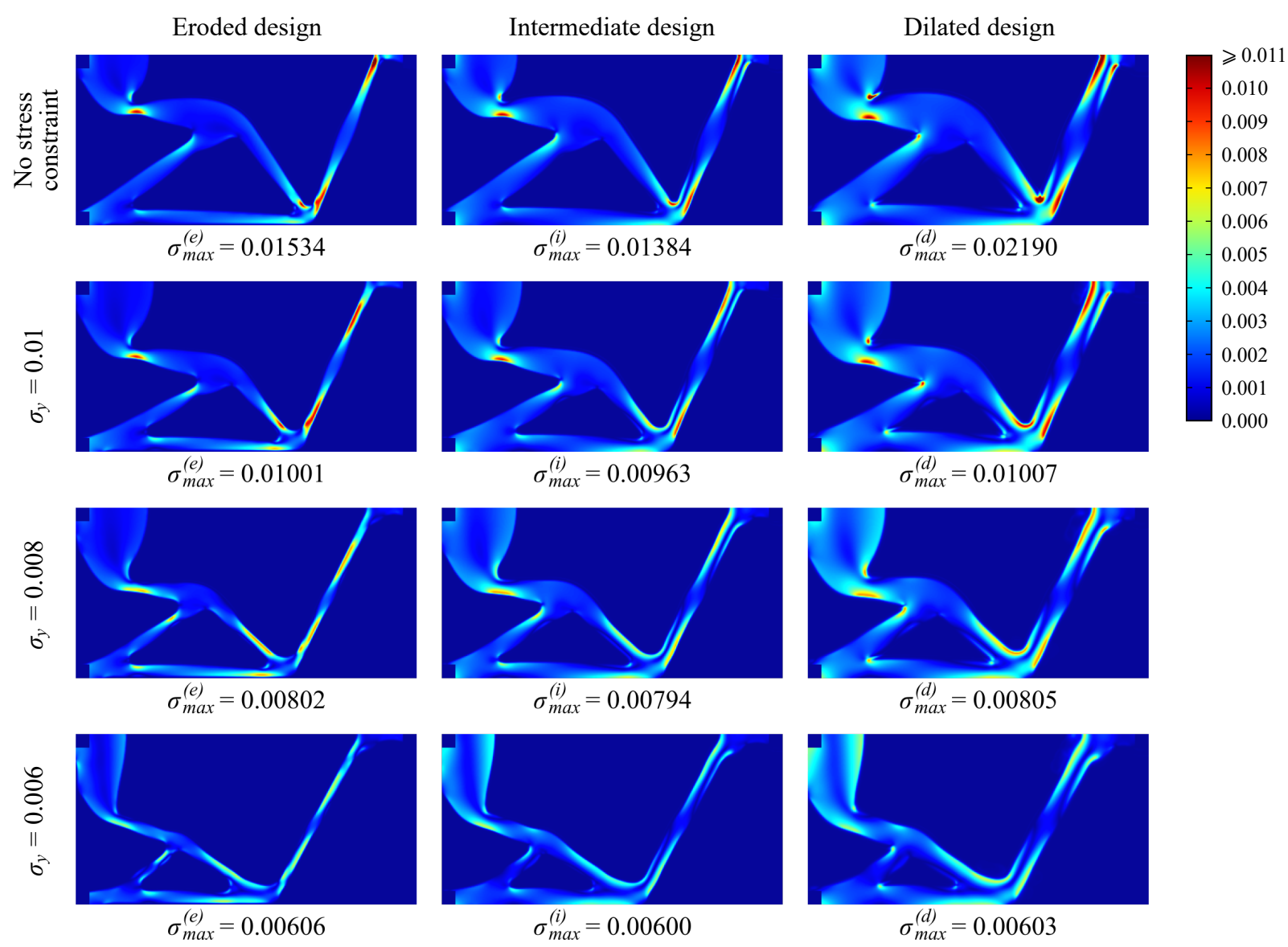

Figure 7: Inverter problem. Von Mises stresses for structures from Figure 6.

intermediate and dilated designs get distributed. It is also clearly verified that optimized designs obtained without considering the stress constraints have some isolated points with high stresses.

Figure 8 shows body-fitted von Mises equivalent stresses relative to eroded, intermediate and dilated topologies. By comparing Figures 7 and 8, one can see good agreement between pixel-based and body-fitted stresses.

Figures 9 and 10 show post-processing graphs of maximum von Mises equivalent stresses and output displacements, respectively, for $\eta \in\left[\eta_{d}, \eta_{e}\right]=[0.25,0.75]$. It is interesting to observe that there is a compromise relation between maximum stresses and output displacements in this case: the smaller the value of $\sigma_{y}$, the smaller the output displacements of the inverter mechanism (in absolute values). When stress constraints are not taken into account, the output displacement of the optimized mechanism is robust with respect to uniform boundary variation. Nevertheless, this robustness is not extended to the maximum von Mises equivalent stress, that exhibits an unpredictable and non-constant behavior for $\eta \in\left[\eta_{d}, \eta_{e}\right]$. In contrast, when stress constraints are considered, the resulting optimized mechanisms exhibit smooth and constant maximum stress response for uniform boundary variations. 


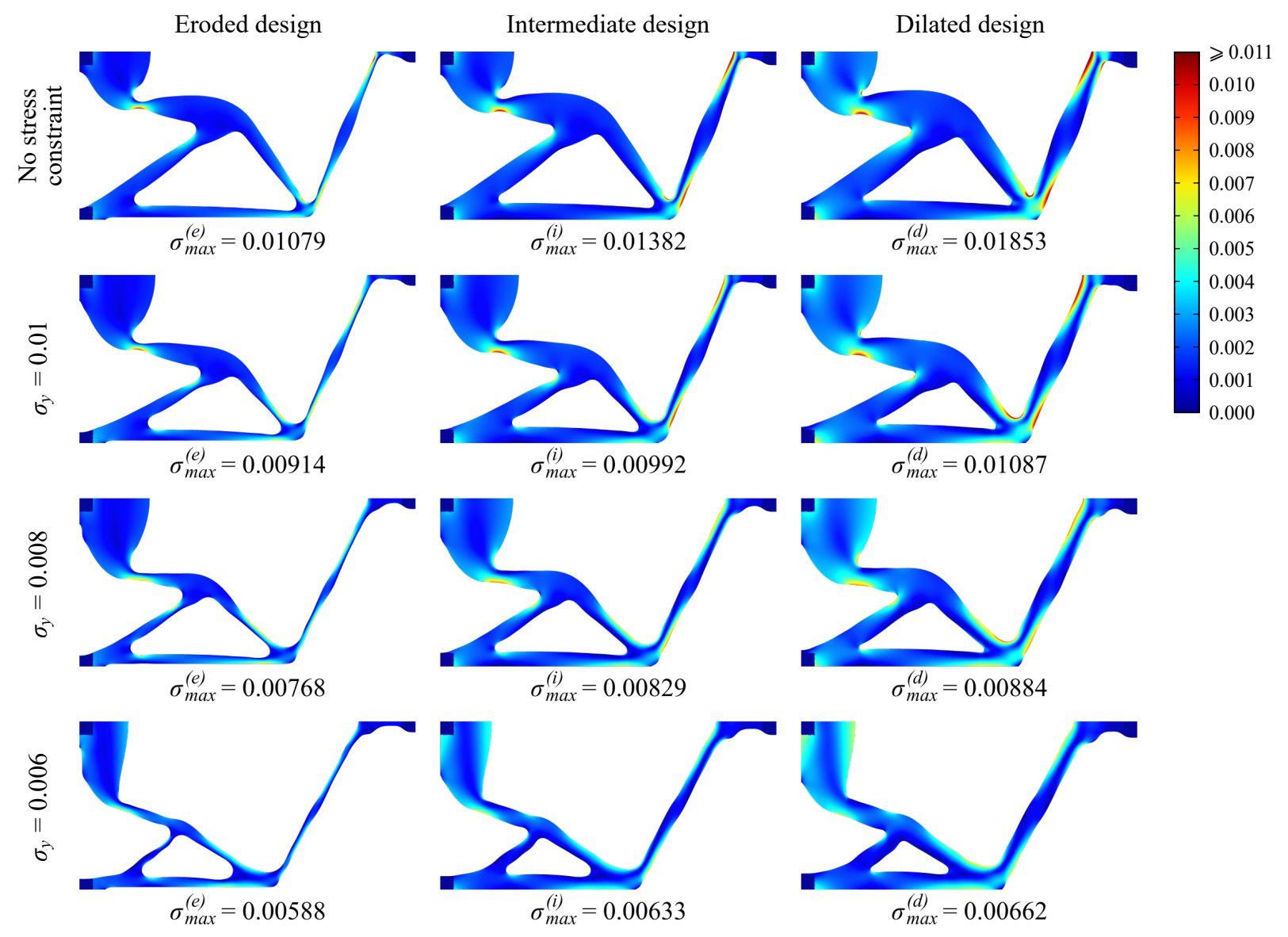

Figure 8: Inverter problem. Body-fitted von Mises stresses for structures from Figure 6.

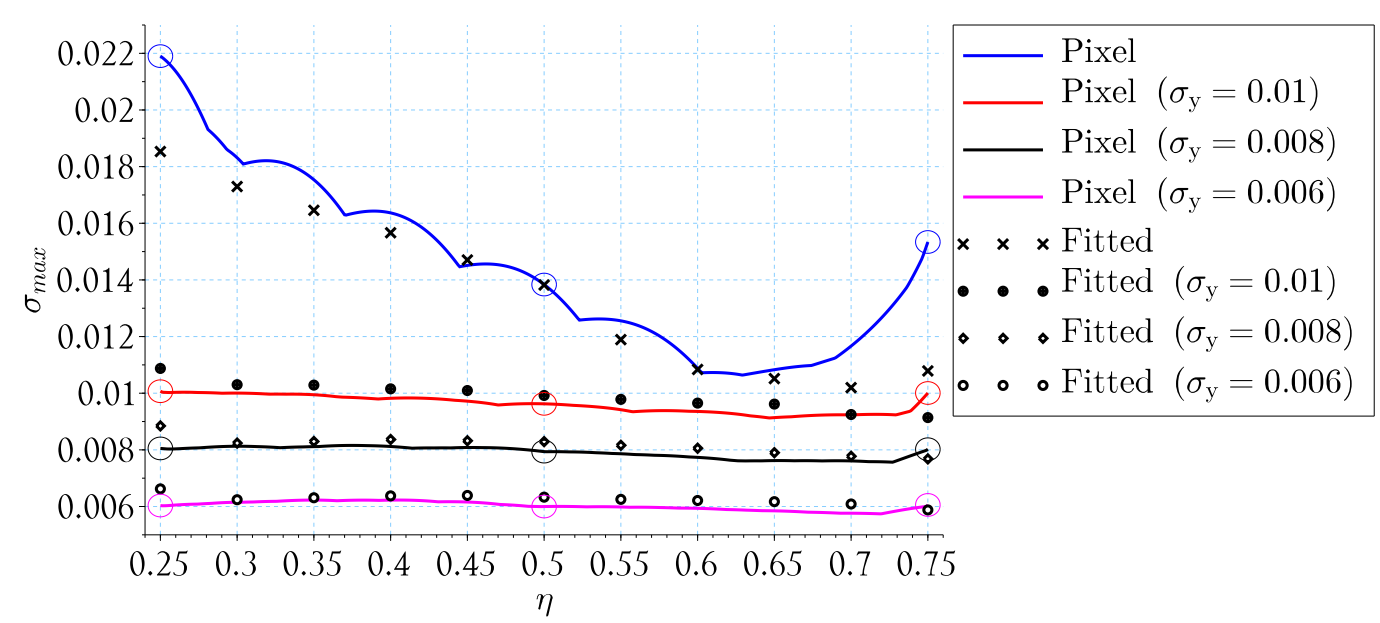

Figure 9: Inverter problem. Maximum von Mises equivalent stresses for $\eta \in[0.25,0.75]$ considering both pixel-based and body-fitted models.

Table 1 shows the maximum stress constraint violation throughout the whole interval $\eta \in\left[\eta_{d}, \eta_{e}\right]$ for both pixelbased and body-fitted models, the maximum difference between the output displacements from pixel-based and 


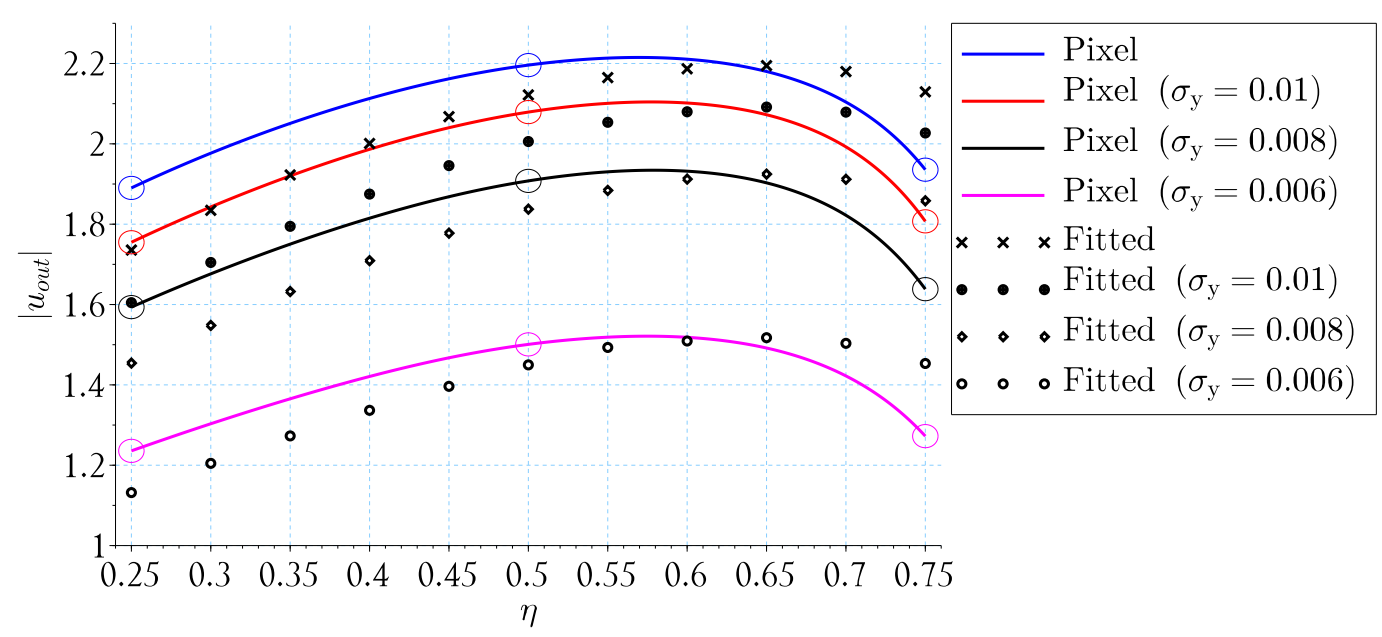

Figure 10: Inverter problem. Output displacements for $\eta \in[0.25,0.75]$ considering both pixel-based and body-fitted models.

body-fitted models, the structural volume of the intermediate topology, the number of iterations until convergence, and the number of objective function evaluations (augmented Lagrangian). The number of iterations is different from the number of function evaluations, since the step length is chosen (in an inexact way), at each iteration of a given optimization subproblem, to ensure the minimization of the augmented Lagrangian. Note, however, that the total number of objective function evaluations is only slightly larger than the number of iterations, since in most iterations the minimization of the augmented Lagrangian is ensured considering the initial estimate for the step length.

Table 1: Inverter problem. Maximum stress constraint violations, maximum differences between output displacements from pixel-based and body-fitted models, volume of intermediate topologies, total number of iterations to satisfy convergence criteria, and number of function evaluations.

\begin{tabular}{|c|c|c|c|c|c|c|c|}
\hline Stress constraint & $\frac{\sigma_{m a x}^{p i x e l}-\sigma_{y}}{\sigma_{y}}$ & $\frac{\sigma_{m a x}^{\text {fitued }}-\sigma_{y}}{\sigma_{y}}$ & $\max$ & 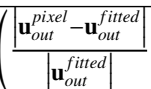 & $V_{f}^{(i)}$ & Iterations & Obj. evaluations \\
\hline Unconstrained & - & - & & $9.10 \%$ & 0.2960 & 500 & 532 \\
\hline$\sigma_{y}=0.01$ & $0.70 \%$ & $8.75 \%$ & & $10.85 \%$ & 0.2793 & 560 & 615 \\
\hline$\sigma_{y}=0.008$ & $1.96 \%$ & $10.56 \%$ & & $11.84 \%$ & 0.2548 & 640 & 722 \\
\hline$\sigma_{y}=0.006$ & $3.79 \%$ & $10.37 \%$ & & $12.44 \%$ & 0.2133 & 534 & 627 \\
\hline
\end{tabular}

It is interesting to observe, in Table 1, that the smaller the value of $\sigma_{y}$, the smaller the volume fraction of the intermediate design $V_{f}^{(i)}$. It is also verified that the volume fraction of the intermediate design does not reach the upper bound, $V_{u p}=0.3$, even when the stress constraint is not considered. This is justified, since we are considering $k_{v}=2$ also in the unconstrained stress problem; in this case the algorithm also sees volume minimization of the dilated design as a design goal, thus preventing the upper volume constraint in being active in some situations. Regarding the maximum stress constraint violations, one can see a maximum value of $10.56 \%$ with respect to the yield stress for $\sigma_{y}=0.008$; and it is interesting to observe, in Figure 9, that the greatest stress constraint violations occur for the dilated design, in this case. The maximum difference between the output displacements is $12.44 \%$, and it occurs for the 
eroded design considering $\sigma_{y}=0.006$. It is shown later, in subsection 4.3.2, for the gripper design problem, that a finer mesh can be employed to reduce both maximum stress constraint violation and maximum difference between the output displacements from pixel-based and body-fitted models.

\subsubsection{Gripper problem}

Figures 11 and 12 show optimized topologies (eroded, intermediate and dilated) and respective pixel-based von Mises equivalent stresses, for the gripper mechanism problem.
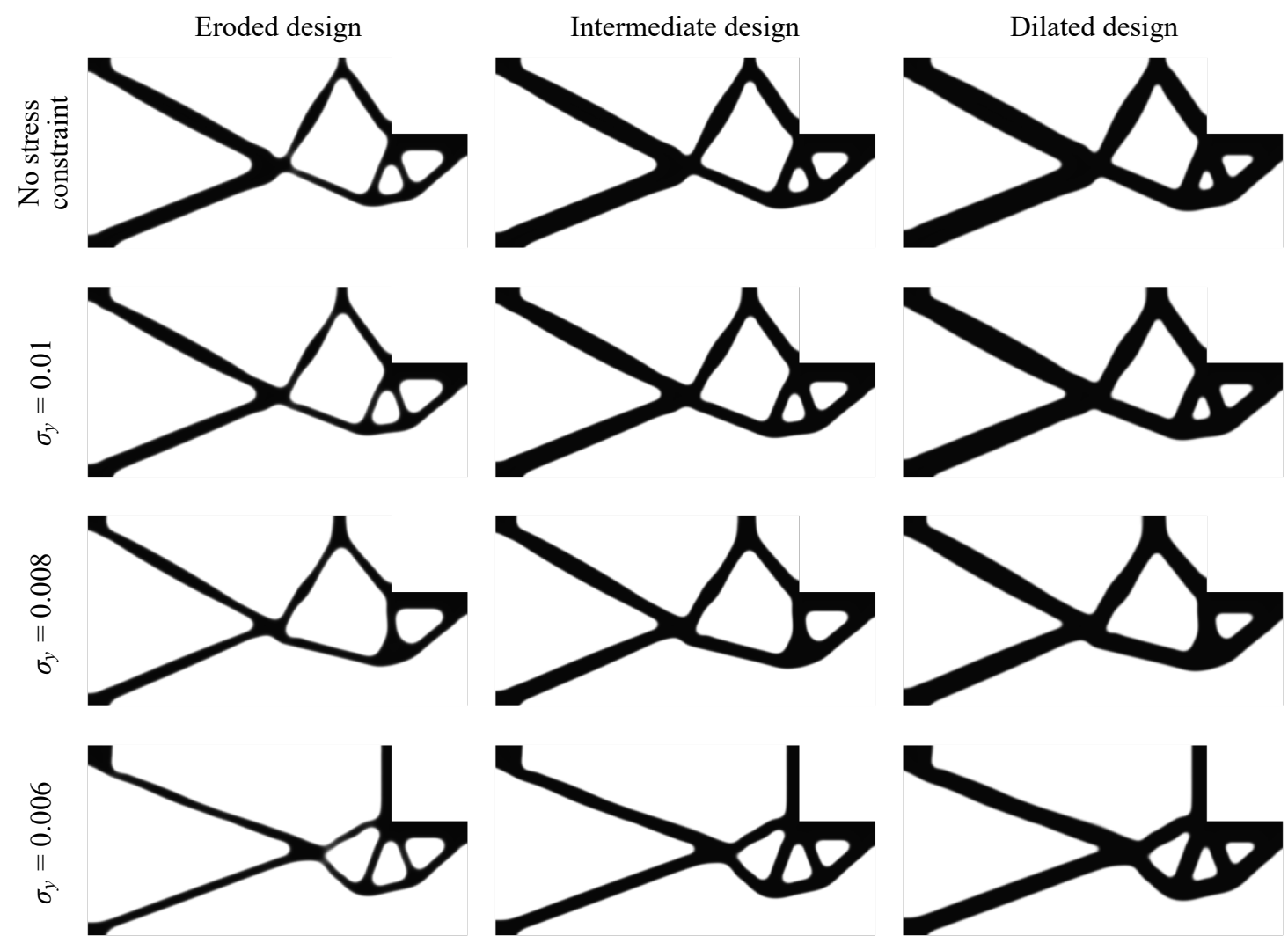

Figure 11: Gripper problem. Optimized topologies for $k_{v}=2$ and four levels of stress constraints: no stress constraints; $\sigma_{y}=0.01 ; \sigma_{y}=0.008$; and $\sigma_{y}=0.006$.

By analyzing Figure 11, one can verify that the smaller the value of $\sigma_{y}$, the thinner the structural members that connect the input port and the support region on the bottom left of the design domain. Regarding the pixel-based von Mises stress fields, Figure 12, one can verify the same behavior as observed before for the inverter problem: the smaller the value of $\sigma_{y}$, the more the stresses become distributed for the optimized designs. Moreover, optimized designs obtained without considering stress constraints have some isolated points with high stresses. Figure 13 shows the body-fitted von Mises equivalent stresses, which exhibit good agreement when compared with the pixel-based stresses shown in Figure 12. 

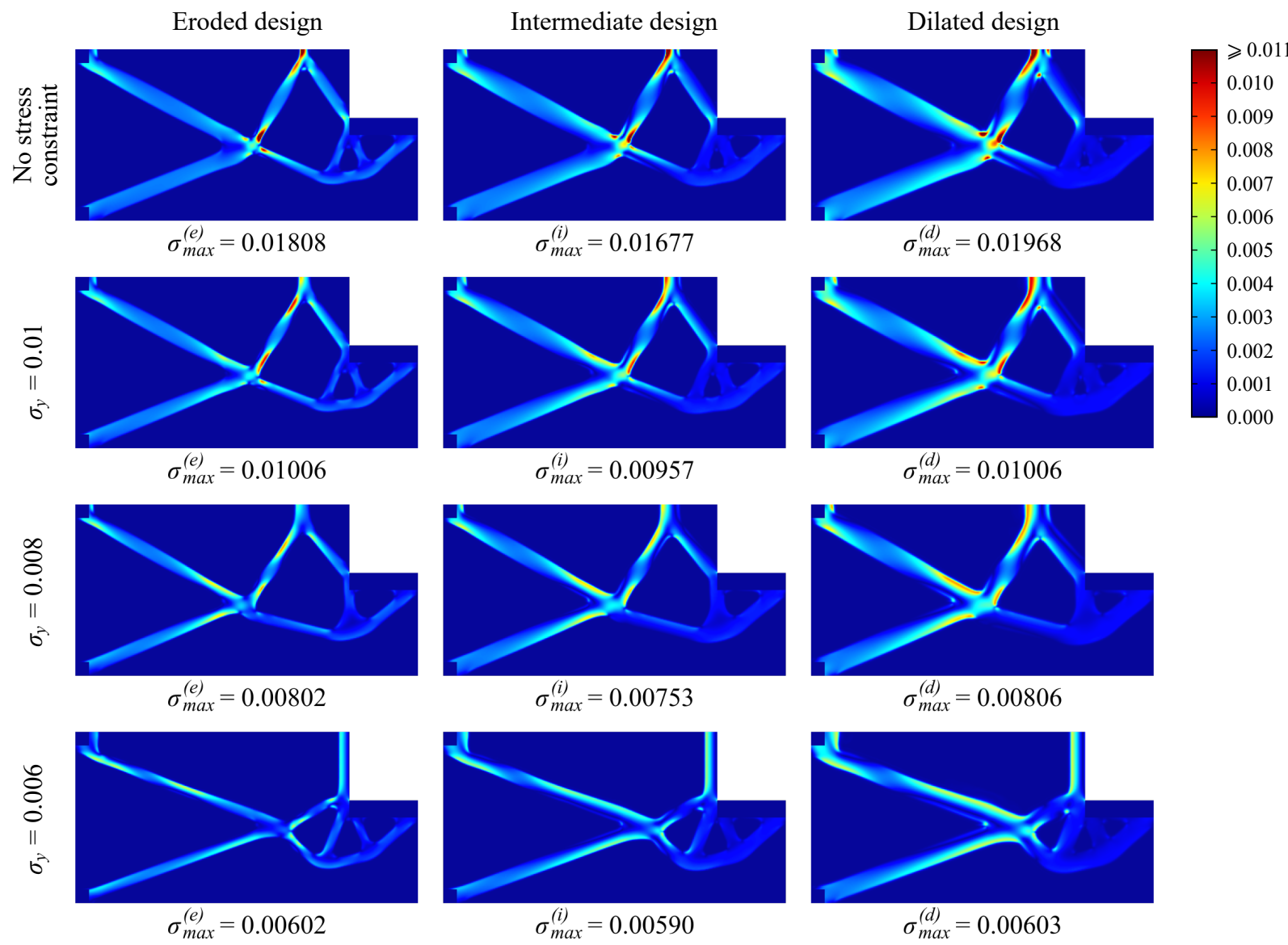

Figure 12: Gripper problem. Von Mises stresses for structures from Figure 11.

Figures 14 and 15 show post-processing graphs of maximum von Mises equivalent stresses and output displacements, respectively, for $\eta \in\left[\eta_{d}, \eta_{e}\right]=[0.3,0.7]$. Regarding the stress responses, one can verify that obtained designs exhibit smooth and almost constant maximum stress response after uniform boundary variation when stress constraints are considered, whereas an unpredictable stress behavior is achieved when no stress constraint is considered. Regarding the displacement responses, one can observe that, the smaller the value of $\sigma_{y}$, the smaller the output displacement of the gripper mechanism (in absolute value), in agreement with previous results achieved for the inverter mechanism problem.

Table 2 shows the maximum stress constraint violation throughout the whole interval $\eta \in\left[\eta_{d}, \eta_{e}\right]$ for both pixelbased and body-fitted models, the maximum difference between the output displacements from pixel-based and body-fitted models, the structural volume of the intermediate topology, the number of iterations until convergence, and the number of objective function evaluations. It is verified that the smaller the value of $\sigma_{y}$, the smaller the volume fraction of the intermediate design $V_{f}^{(i)}$, in agreement with the inverter results shown in Table 1. One can also observe 

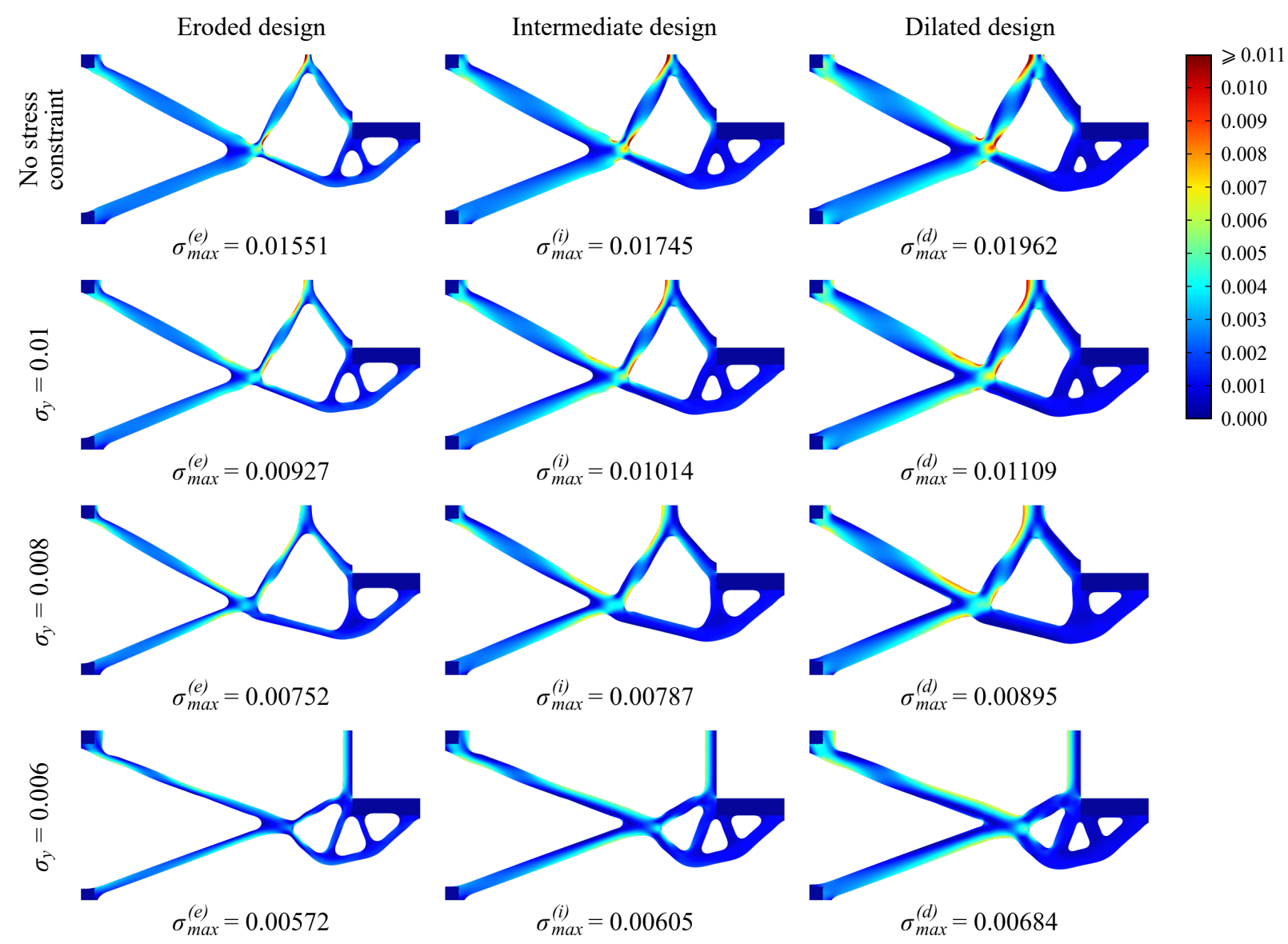

Figure 13: Gripper problem. Body-fitted von Mises stresses for structures from Figure 11.

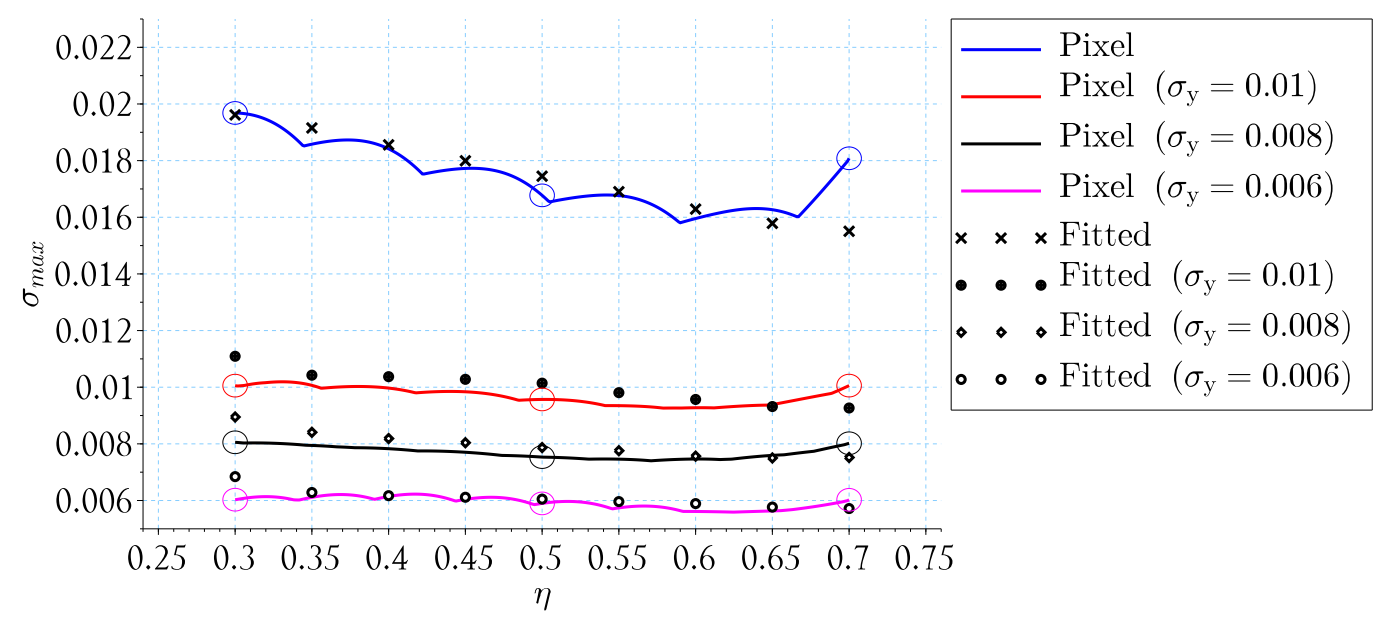

Figure 14: Gripper problem. Maximum von Mises equivalent stresses for $\eta \in[0.3,0.7]$ considering both pixel-based and body-fitted models.

that the volume constraint is not active in these cases, as also observed earlier for the inverter mechanism problem. Regarding the maximum stress constraint violations, one can see a maximum value of $14.05 \%$ for solution obtained 


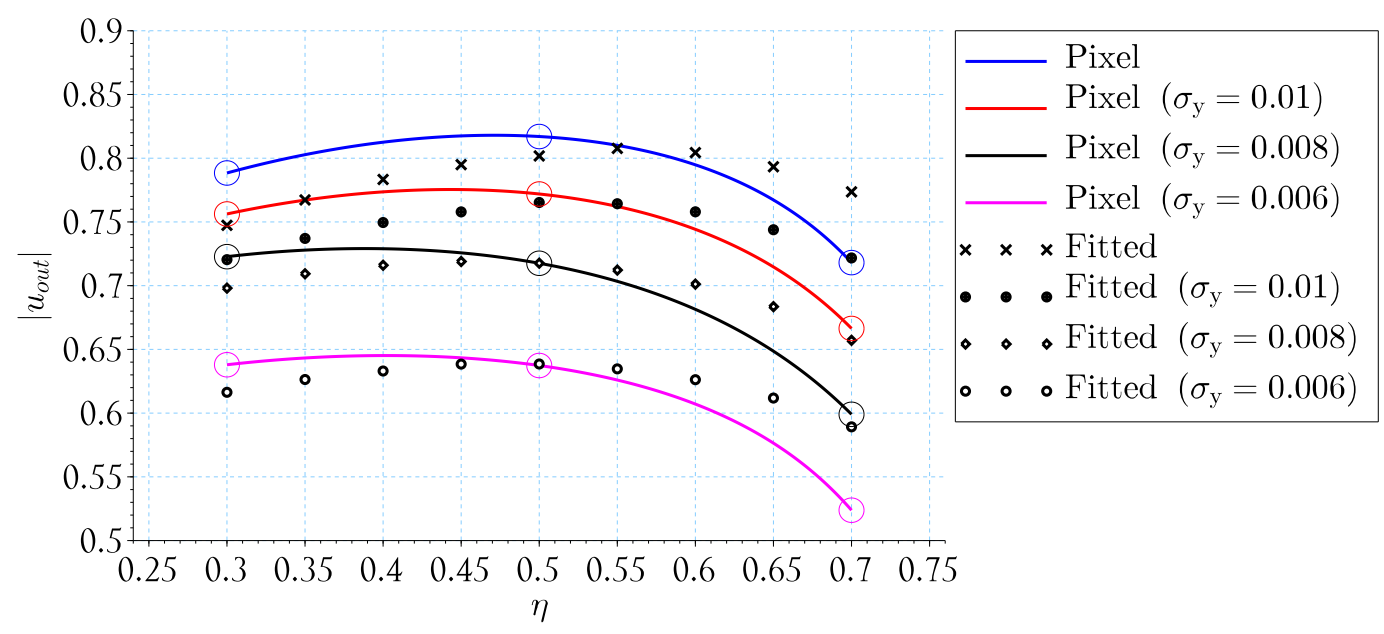

Figure 15: Gripper problem. Output displacements for $\eta \in[0.3,0.7]$ considering both pixel-based and body-fitted models.

with $\sigma_{y}=0.006$. The maximum stress constraint violations occur in the dilated design in the gripper problem. The maximum difference between the output displacements is $11.10 \%$, and it occurs for the eroded design considering $\sigma_{y}=0.006$.

Table 2: Gripper problem. Maximum stress constraint violations, maximum differences between output displacements from pixel-based and body-fitted models, volume of intermediate topologies, total number of iterations to satisfy convergence criteria, and number of function evaluations.

\begin{tabular}{lcccccc}
\hline Stress constraint & $\frac{\sigma_{\text {max }}^{\text {pixal }}-\sigma_{y}}{\sigma_{y}}$ & $\frac{\sigma_{\text {max }}^{\text {fitud }}-\sigma_{y}}{\sigma_{y}}$ & $\max \left(\frac{\left|\mathbf{p}_{\text {out }}^{\text {pixel }}-\mathbf{u}_{\text {out }}^{\text {fited }}\right|}{\left|\mathbf{u}_{\text {out }}^{\text {fited }}\right|}\right)$ & $V_{f}^{(i)}$ & Iterations & Obj. evaluations \\
\hline Unconstrained & - & - & $7.18 \%$ & 0.2542 & 500 & 541 \\
$\sigma_{y}=0.01$ & $1.91 \%$ & $10.92 \%$ & $7.67 \%$ & 0.2394 & 500 & 546 \\
$\sigma_{y}=0.008$ & $0.69 \%$ & $11.85 \%$ & $8.83 \%$ & 0.2213 & 560 & 629 \\
$\sigma_{y}=0.006$ & $3.75 \%$ & $14.05 \%$ & $11.10 \%$ & 0.2084 & 500 & 609 \\
\hline
\end{tabular}

\subsection{Additional numerical results}

This subsection presents additional numerical results, to provide additional insight. Two additional studies are performed: in subsection 4.3.1, the deterministic stress-constrained compliant mechanism design approach is compared with the proposed robust stress-constrained approach; in subsection 4.3.2, a mesh dependence study is performed. 


\subsubsection{Comparison between deterministic and robust stress-constrained approaches}

The standard deterministic stress-constrained approach for compliant mechanism design can be written as [2]

$$
\begin{array}{cll}
\underset{\boldsymbol{\rho}}{\operatorname{Min} .} & u_{\text {out }}(\overline{\boldsymbol{\rho}}) \\
& & \\
\text { s. t. } & V_{f}(\overline{\boldsymbol{\rho}}) \leqslant V_{u p} & \\
& \frac{\sigma_{\text {eq }}^{(k)}(\overline{\boldsymbol{\rho}})}{\sigma_{y}}-1 \leqslant 0 & k=1,2, \ldots, N_{k} \\
& \mathbf{K}(\overline{\boldsymbol{\rho}}) \mathbf{U}(\overline{\boldsymbol{\rho}})=\mathbf{F} & \\
& 0 \leqslant \rho_{e} \leqslant 1 \quad & e=1,2, \ldots, N_{e}
\end{array}
$$

In this formulation, only one field of physical relative densities $\bar{\rho}$ is considered. In this paper, the solution is obtained by employing the threshold projection function, Equation (2), for $\eta=0.5$.

Both inverter and gripper design problems are solved with the deterministic formulation, considering the same input data as defined in the beginning of section 4 , and compared with results presented in subsection 4.2. The only difference is in the choice of both initial and maximum penalization parameters for the stress constraints (associated with the augmented Lagrangian method), which are three times larger than those defined for the robust case, such that: $r^{(1)}=3 / N_{e}$ and $r_{\max }=3 \times 10^{5} / N_{e}$; since in this case we have only one set of stress constraints. Both problems are solved for $\sigma_{y}=0.006$, which is the smallest yield stress value employed in this paper. Optimized designs are post-processed with pixel-based and body-fitted approaches for $\eta \in\left[\eta_{d}, \eta_{e}\right]$.

Figures 17, 18 and 19 show optimized results (deterministic and robust topologies with respective pixel-based and body-fitted von Mises stresses), post-processing graphs of von Mises stresses and post-processing graphs of output displacements, for the inverter problem. Figures 20, 21 and 22 show these same results for the gripper problem. However, in the deterministic case, designs with disconnected structural members are obtained for $\eta>0.6$, considering the inverter design problem, and $\eta>0.55$, for the gripper problem, as shown in Figure 16; thus, the respective post-processing graphs show the numerical responses up to these values of $\eta$ only.

Since both deterministic solutions become disconnected when the post-processing schemes are employed for too large values of $\eta$, these solutions are clearly non-robust (both maximum stresses and output displacements) with respect to uniform boundary variations resulting from erosion operations.

Considering now the intervals for which physically reasonable results are obtained, i.e., $\eta \in[0.25,0.6]$, for the inverter case, and $\eta \in[0.3,0.55]$, for the gripper case, one can draw two important conclusions: 1) deterministic solutions totally outperform the robust ones regarding the output displacements; and 2) the maximum von Mises stresses of deterministic solutions are clearly non-robust with respect to uniform boundary variations. 


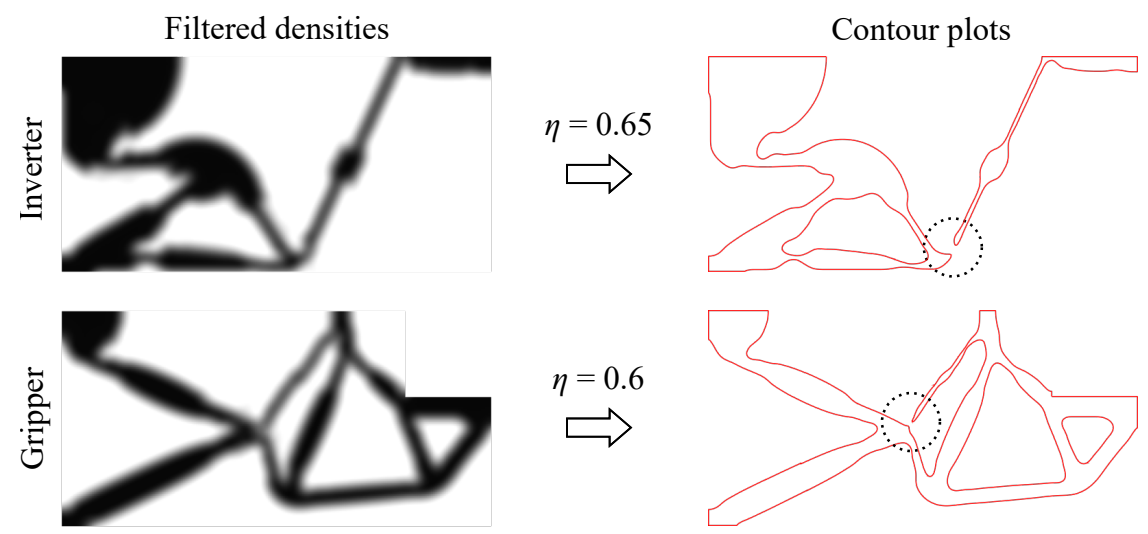

Figure 16: Deterministic results. Optimized filtered densities and respective contour plots for $\eta=0.65$ (inverter) and $\eta=0.6$ (gripper). Disconnected regions are highlighted by dashed circles.
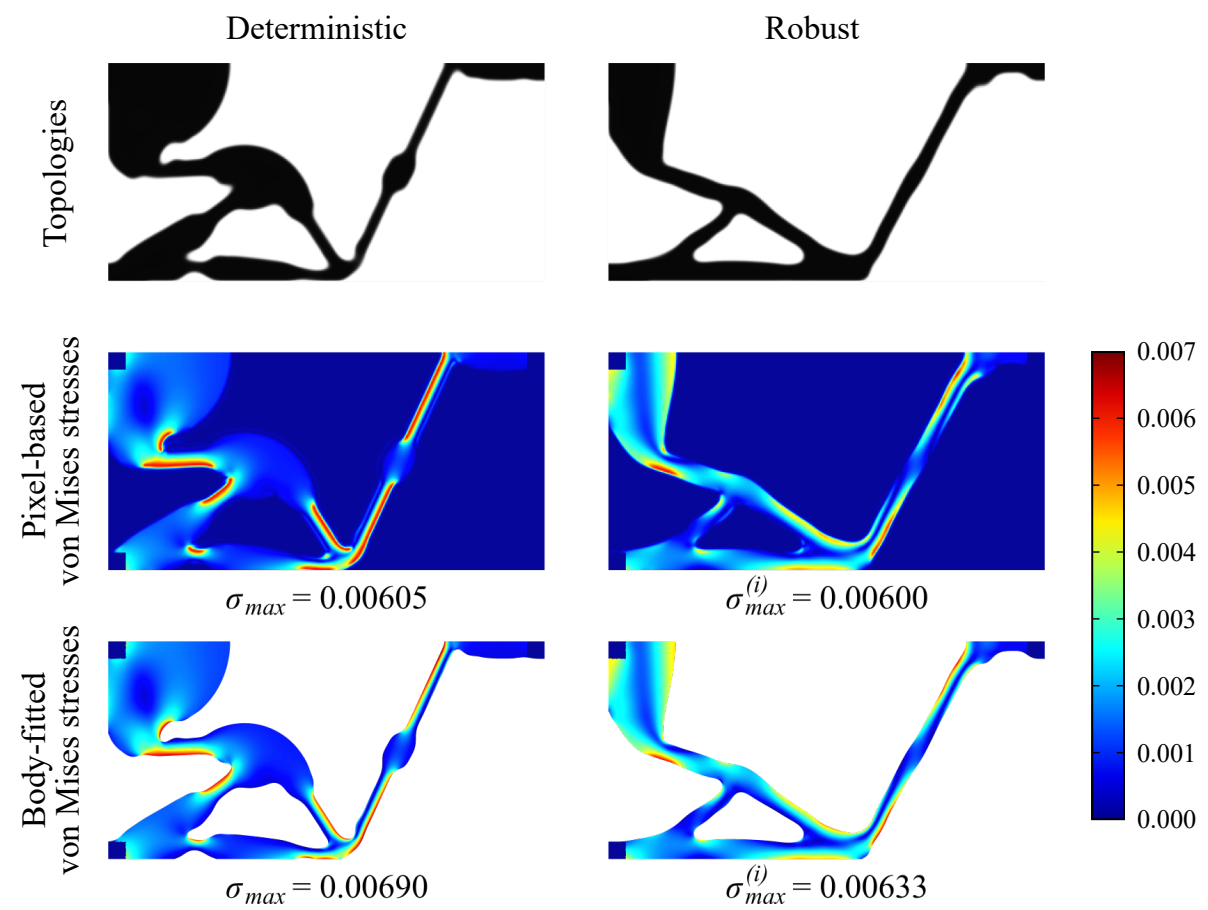

Figure 17: Inverter problem. Optimized topologies and respective pixel-based and body-fitted stresses, for $\sigma_{y}=0.006$, considering both deterministic and robust stress-constrained approaches.

The reduced performances regarding output displacements are justified, since the robust stress-constrained approach has additional challenge regarding stress feasibility, which should be ensured for three distinct fields of relative densities. The deterministic stress-constrained approach, on the other hand, works by applying the stress constraints over one field of relative densities only, so that there is more freedom to distribute material in a way the minimization of the output displacement is prioritized, at the cost of obtaining highly non-robust stress behavior given uniform boundary variations. 


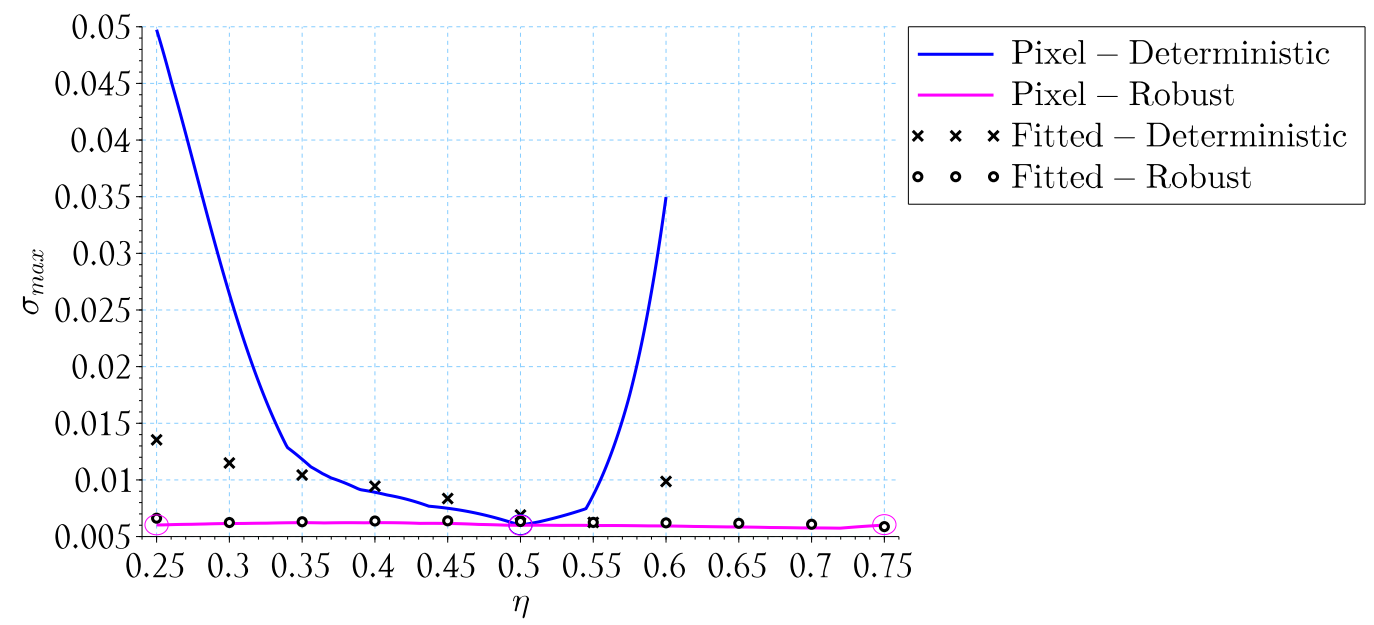

Figure 18: Inverter problem. Maximum von Mises equivalent stresses for $\eta \in[0.25,0.75]$ (robust) and $\eta \in[0.25,0.6]$ (deterministic), considering both pixel-based and body-fitted models.

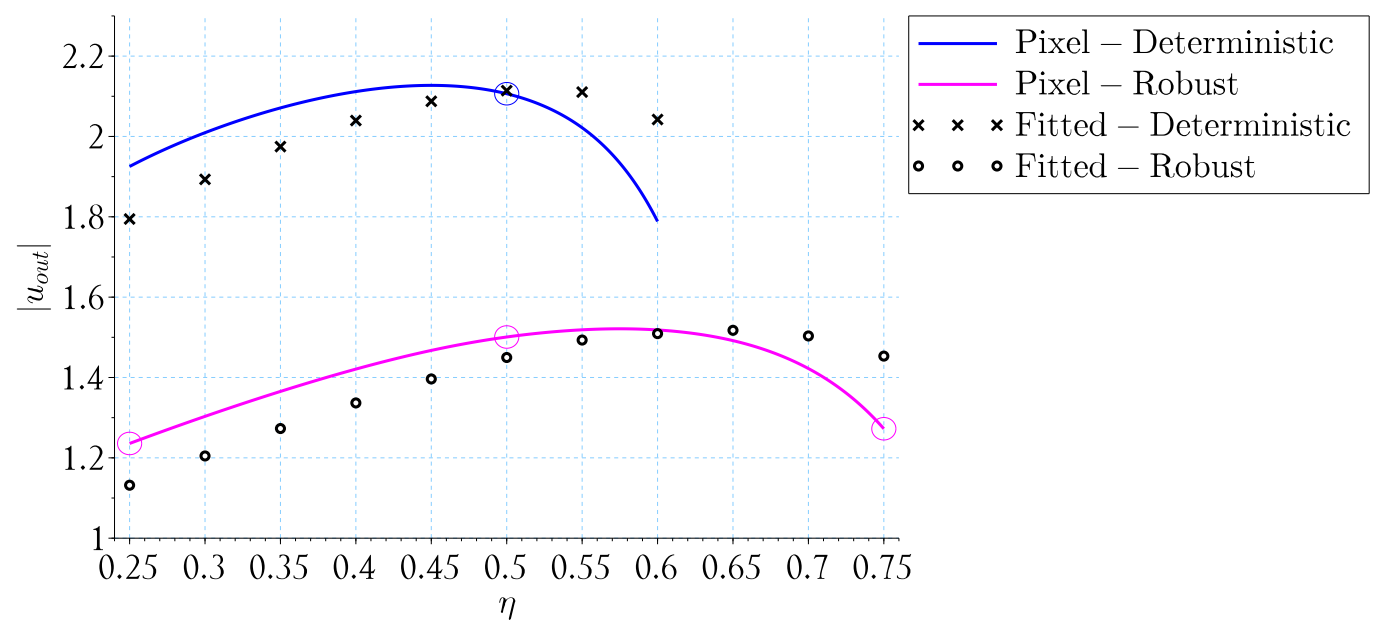

Figure 19: Inverter problem. Output displacements for $\eta \in[0.25,0.75]$ (robust) and $\eta \in[0.25,0.6]$ (deterministic), considering both pixel-based and body-fitted models.

\subsubsection{Mesh dependence study}

This subsection addresses the gripper mechanism problem only. The problem is solved for two distinct situations considering more refined meshes than before: 1) $N_{e}=294,400$ and $R=2.8$; and 2) $N_{e}=294,400$ and $R=1.4$. These meshes correspond to $800 \times 400$ grids, disregarding the elements inside the void square region on the upper right of the design domain. The difference between both cases is in the radius of the filter $R$, which has half size in the second case. Both cases are solved for $\sigma_{y}=0.006$, considering same data as defined in the beginning of section 4 . The only difference is in parameter $\beta$, which is updated from $\beta^{(1)}=1$ to $\beta_{\max }=\beta_{\text {lim }} / 2=22.4$ in the first case, and from $\beta^{(1)}=0.5$ to $\beta_{\max }=\beta_{\text {lim }} / 2=11.2$ in the second case, where a smaller $R$ is employed. 

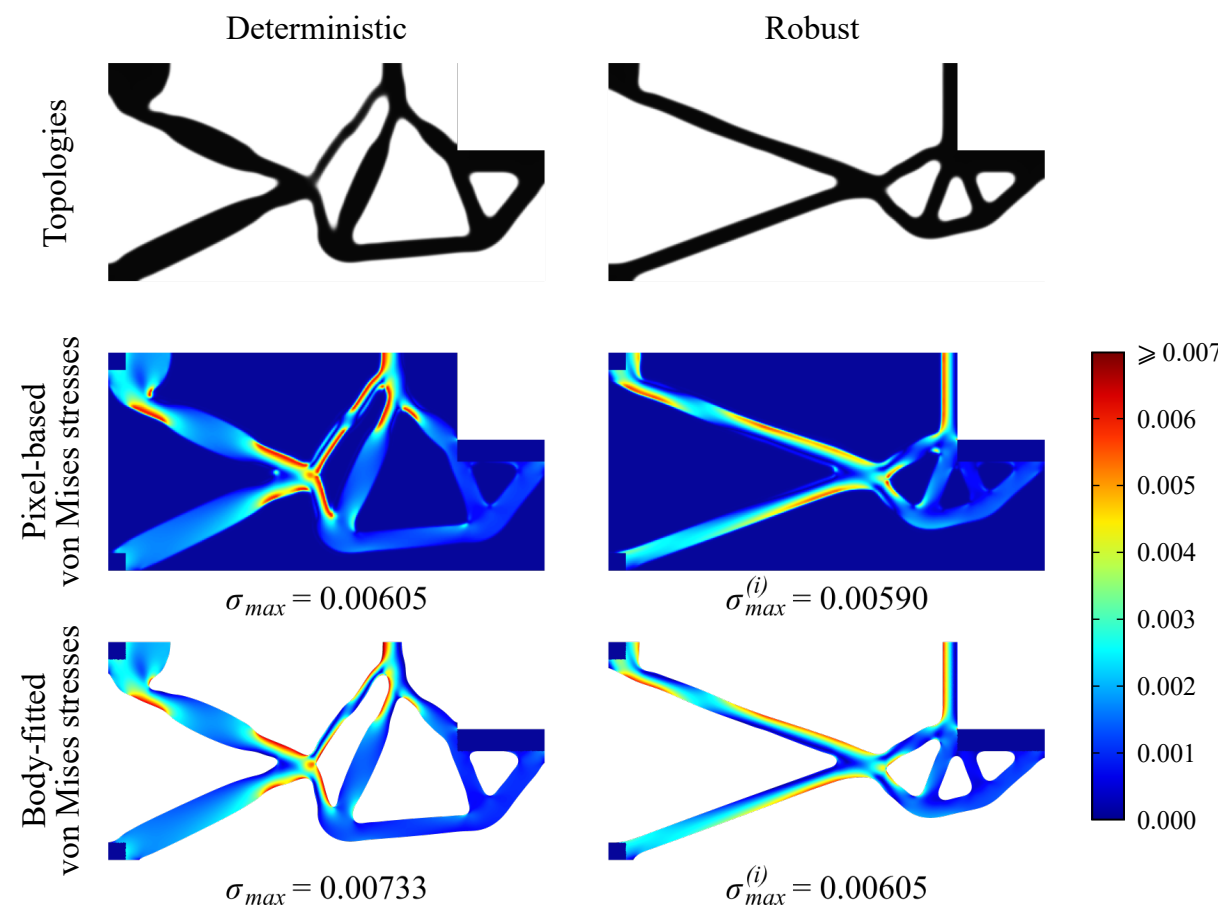

Figure 20: Gripper problem. Optimized topologies and respective pixel-based and body-fitted stresses, for $\sigma_{y}=0.006$, considering both deterministic and robust stress-constrained approaches.

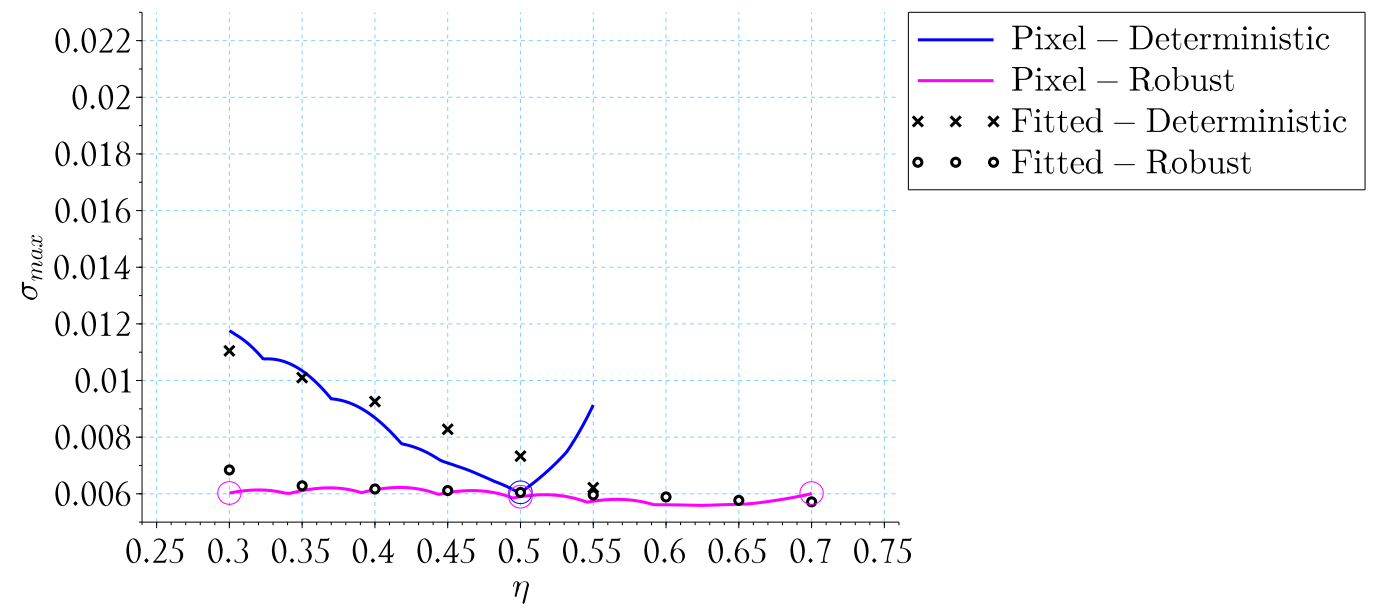

Figure 21: Gripper problem. Maximum von Mises equivalent stresses for $\eta \in[0.3,0.7]$ (robust) and $\eta \in[0.3,0.55]$ (deterministic), considering both pixel-based and body-fitted models.

Figures 23 and 24 show robust results and post-processing graphs, respectively. Obtained results are shown for both gripper design cases considering $N_{e}=294,400$, and also for the solution presented in subsection 4.2 where a coarser mesh of $N_{e}=73,600$ is employed.

By analyzing Figure 23, one can verify that results for $R=2.8$ present the same topology, with slight differences 


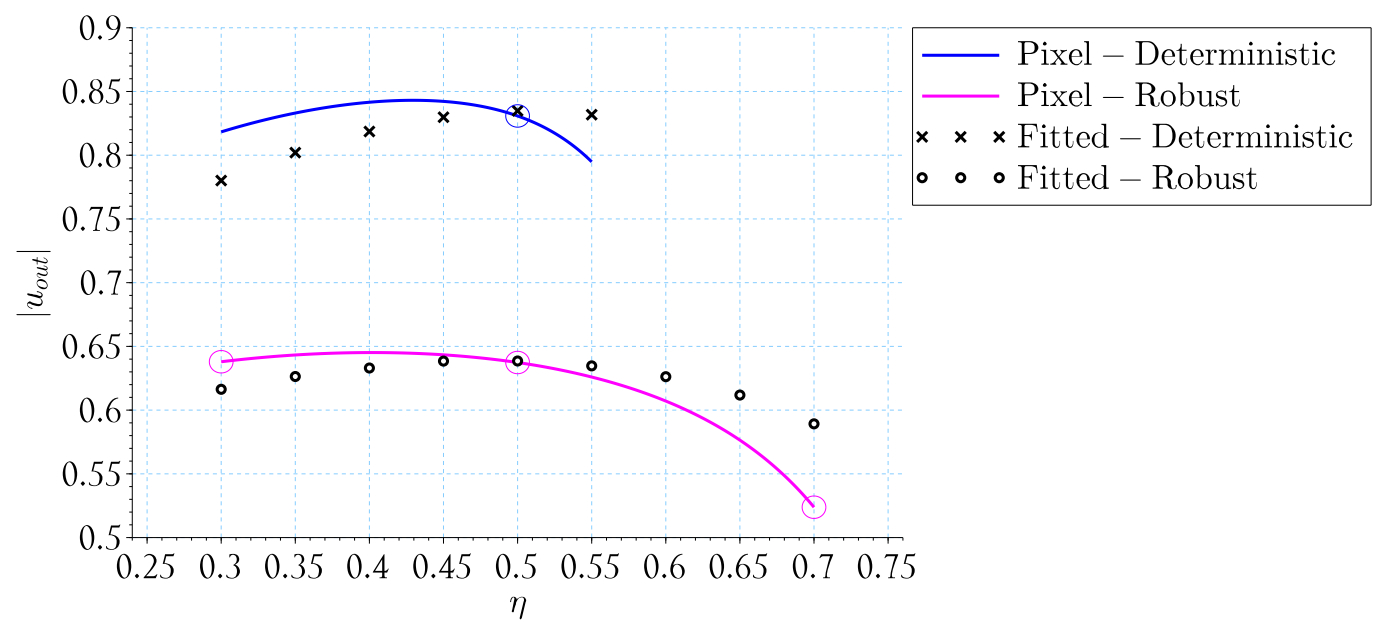

Figure 22: Gripper problem. Output displacements for $\eta \in[0.3,0.7]$ (robust) and $\eta \in[0.3,0.55]$ (deterministic), considering both pixel-based and body-fitted models.
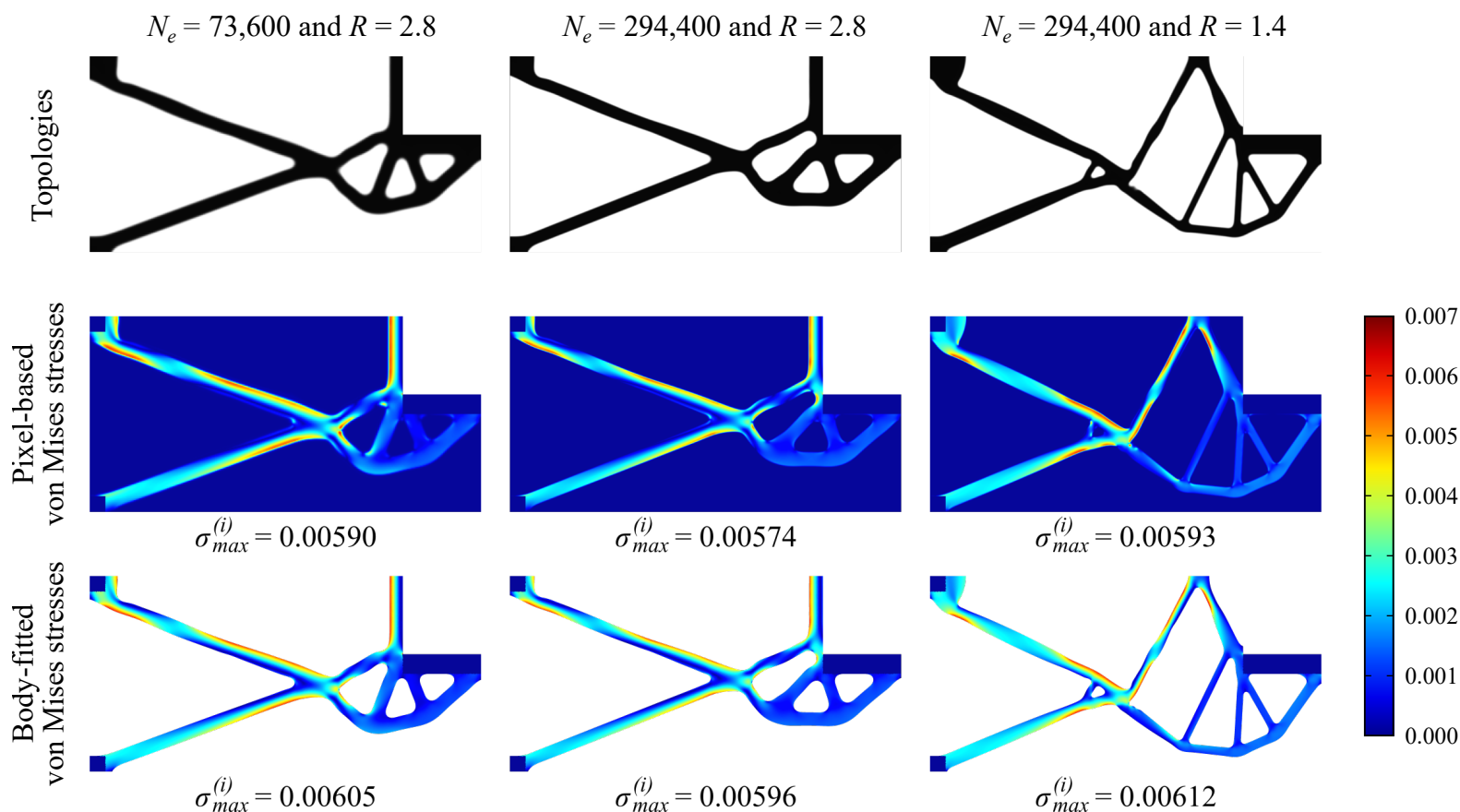

Figure 23: Gripper problem. Optimized robust topologies and respective pixel-based and body-fitted stresses, for $\sigma_{y}=0.006$, considering different number of elements $N_{e}$ and filter's radius $R$.

in shape. For $R=1.4$, on the other hand, a very different topology is obtained, with thinner reinforcements. This is justified, in this case, since the value of $R$ is directly related to a minimum length scale when the robust approach is employed, as discussed in [8]. It is interesting to verify, in Figure 24, that the solution for $R=1.4$ outperforms solutions for $R=2.8$ regarding the output displacements. This is justified, since the smaller the value of $R$, the smaller the difference between dilated and eroded topologies (considering fixed values for $\eta_{d}$ and $\eta_{e}$ ); hence, the smaller the 

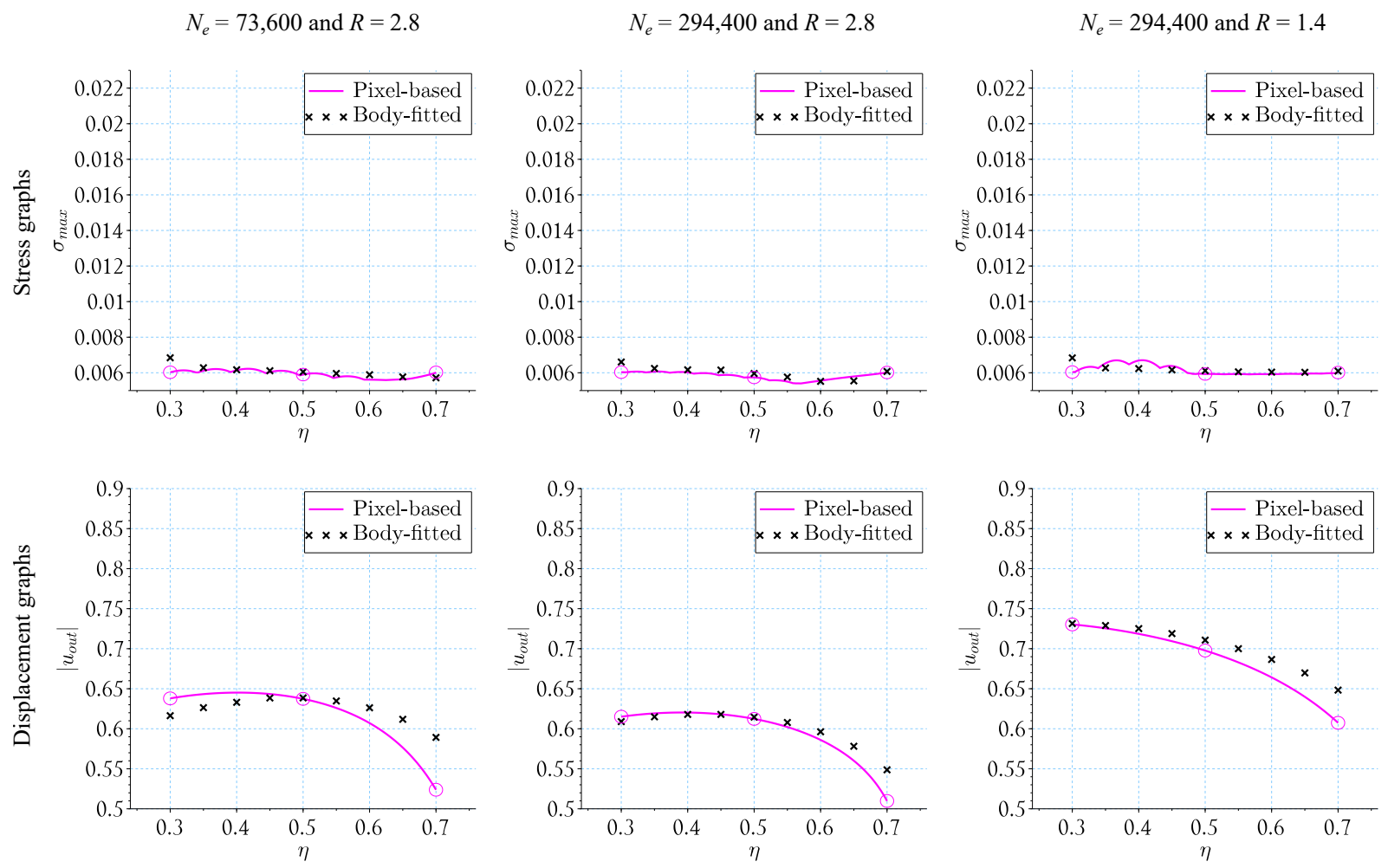

Figure 24: Gripper problem. Post-processing graphs of maximum stresses and output displacements for problems from Figure 23.

robustness of the mechanism with respect to uniform boundary variation; thus, the mechanism gets more freedom to distribute material in a way output displacement minimization is prioritized.

By comparing both results obtained for $R=2.8$, we noticed slight improvement, with mesh refinement, regarding stress feasibility considering the most critical stress models: from 3.75\% (pixel) and $14.05 \%$ (fitted), as shown in Table 2 for $N_{e}=73,600$, to $1.17 \%$ (pixel) and $10.09 \%$ (fitted), for $N_{e}=294,400$; and regarding the maximum difference between output displacements of pixel-based and body-fitted models: from $11.10 \%$, for $N_{e}=73,600$, to $7.09 \%$, for $N_{e}=294,400$.

\section{Concluding remarks}

This work has proposed and investigated a robust design formulation to topology optimization of manufacturing uncertainty tolerant compliant mechanisms, considering stress constraints. Several numerical experiments were performed in order to investigate applicability of the proposed approach. Optimized results were post-processed with both pixel-based and body-fitted finite element models. Some concluding remarks are drawn:

1. The proposed robust approach was able to provide compliant mechanisms that have maximum von Mises stresses 
that are insensitive to uniform boundary variations, for different yield stress values.

2. When solving the robust problem without stress constraints, a very unpredictable stress behavior was observed, considering both inverter and gripper problems, indicating the traditional robust approach is not able to ensure stress robustness with respect to uniform boundary variations, although it does provide much better design than without the robust approach.

3. A comparison between deterministic and robust stress-constrained approaches was performed: robust topologies are quite different than deterministic counterparts. It was shown that the deterministic approach provides optimized solutions for a given field of physical relative densities only, and slight boundary variations cause strong stress constraint violation.

4. A mesh refinement study was performed. It was observed that for a fixed filter radius, a finer mesh can be employed to improve stress feasibility considering both pixel-based and body-fitted stress models. Considering the problem with fixed mesh and different filtering radius, it was demonstrated that a smaller filter radius can be employed to achieve a compliant mechanism with larger output displacements, at the cost of less robustness with respect to uniform boundary variations.

Finally, it is important to mention that a compromise relation between output displacements and maximum von Mises stresses was verified: the smaller the yield stress considered during the optimization process, the smaller the output displacements (in absolute values) of the resulting mechanism.

\section{Appendix: Sensitivity analysis}

This section develops the sensitivity analysis to evaluate the derivative of the augmented Lagrangian function $L$ with respect to a design variable $\rho_{m}$, in order to perform the optimization with gradient-based algorithm.

The augmented Lagrangian function, Equation (10), can be properly rewritten in order to facilitate sensitivity analysis by the adjoint technique:

$$
L=L_{V}+L_{u \sigma}
$$

where $L_{V}$ is the term associated with the volume fraction of the dilated design

$$
L_{V}=k_{v} V_{f}\left(\overline{\boldsymbol{\rho}}^{(d)}\right)+\frac{r_{u p}}{2}\left\langle\frac{\mu_{u p}}{r_{u p}}+\frac{V_{f}\left(\overline{\boldsymbol{\rho}}^{(d)}\right)}{V_{u p}^{(d)}}-1\right\rangle^{2}+\frac{r_{\text {low }}}{2}\left\langle\frac{\mu_{\text {low }}}{r_{\text {low }}}-\frac{V_{f}\left(\overline{\boldsymbol{\rho}}^{(d)}\right)}{V_{\text {low }}^{(d)}}+1\right\rangle^{2},
$$


and $L_{u \sigma}$ is the term associated with both the output displacements and stress constraints

$$
L_{u \sigma}=\frac{k_{\text {in }}}{f_{\text {in }}} u_{\text {out }}^{K S}\left(\overline{\boldsymbol{\rho}}^{(e)}, \overline{\boldsymbol{\rho}}^{(i)}, \overline{\boldsymbol{\rho}}^{(d)}\right)+\frac{r}{2} \sum_{k=1}^{N_{k}} \sum_{j \in S}\left\langle\frac{\mu_{k}^{(j)}}{r}+\frac{\sigma_{e q}^{(k)}\left(\overline{\boldsymbol{\rho}}^{(j)}\right)}{\sigma_{y}}-1\right\rangle^{2}+\sum_{j \in S} \boldsymbol{\lambda}_{(j)}^{T}\left(\mathbf{K}\left(\overline{\boldsymbol{\rho}}^{(j)}\right) \mathbf{U}\left(\overline{\boldsymbol{\rho}}^{(j)}\right)-\mathbf{F}\right),
$$

where $S=\{e, i, d\}$ is the set which contains dilated, intermediate and eroded fields of physical relative densities, and $\lambda_{(j)}$ are arbitrary vectors, since $\mathbf{K}\left(\overline{\boldsymbol{\rho}}^{(j)}\right) \mathbf{U}\left(\overline{\boldsymbol{\rho}}^{(j)}\right)-\mathbf{F}=\mathbf{0}$.

The derivative of the augmented Lagrangian function can be computed through a chain rule, as described in [25], in order to perform optimization in the non-physical design space. Once the term $L_{V}$ is dependent on the dilated densities only, one can write

$$
\frac{\partial L}{\partial \rho_{m}}=\sum_{n \in \vartheta_{m}} \frac{\partial L_{V}}{\partial \bar{\rho}_{n}^{(d)}} \frac{\partial \bar{\rho}_{n}^{(d)}}{\partial \tilde{\rho}_{n}} \frac{\partial \tilde{\rho}_{n}}{\partial \rho_{m}}+\sum_{j \in S} \sum_{n \in \vartheta_{m}} \frac{\partial L_{u \sigma}}{\partial \bar{\rho}_{n}^{(j)}} \frac{\partial \bar{\rho}_{n}^{(j)}}{\partial \tilde{\rho}_{n}} \frac{\partial \tilde{\rho}_{n}}{\partial \rho_{m}}
$$

where

$$
\frac{\partial L_{V}}{\partial \bar{\rho}_{n}^{(d)}}=\frac{V_{n}}{V_{\text {domain }}}\left[k_{v}+\left\langle\mu_{u p}+r_{u p}\left(\frac{V_{f}\left(\overline{\boldsymbol{\rho}}^{(d)}\right)}{V_{u p}^{(d)}}-1\right)\right\rangle \frac{1}{V_{u p}^{(d)}}-\left\langle\mu_{\text {low }}+r_{\text {low }}\left(-\frac{V_{f}\left(\overline{\boldsymbol{\rho}}^{(d)}\right)}{V_{\text {low }}^{(d)}}+1\right)\right\rangle \frac{1}{V_{\text {low }}^{(d)}}\right]
$$

is the derivative of Equation (.2),

$$
\frac{\partial \bar{\rho}_{n}^{(j)}}{\partial \tilde{\rho}_{n}}=\frac{\beta\left(\operatorname{sech}\left(\beta\left(\tilde{\rho}_{n}-\eta_{j}\right)\right)\right)^{2}}{\tanh \left(\beta \eta_{j}\right)+\tanh \left(\beta\left(1-\eta_{j}\right)\right)}
$$

is the derivative of Equation (2), and

$$
\frac{\partial \tilde{\rho}_{n}}{\partial \rho_{m}}=\frac{w\left(\mathbf{x}_{m}\right) V_{m}}{\sum_{o \in \vartheta_{n}} w\left(\mathbf{x}_{o}\right) V_{o}}
$$

is the derivative of Equation (3).

One can show that the derivative of $L_{u \sigma}$, Equation (.3), with respect to a physical relative density $\bar{\rho}_{n}^{(j)}$, is computed as (see [17] for further insight on the stress derivative):

$$
\begin{aligned}
\frac{\partial L_{u \sigma}}{\partial \bar{\rho}_{n}^{(j)}}= & \sum_{k=1}^{N_{k}} h_{k}^{(j)} \frac{\partial f_{\sigma}\left(\bar{\rho}_{k}^{(j)}\right)}{\partial \bar{\rho}_{n}^{(j)}} \hat{\sigma}_{e q}^{(k)}\left(\overline{\boldsymbol{\rho}}^{(j)}\right)+\boldsymbol{\lambda}_{(j)}^{T} \frac{\partial \mathbf{K}\left(\overline{\boldsymbol{\rho}}^{(j)}\right)}{\partial \bar{\rho}_{n}^{(j)}} \mathbf{U}\left(\overline{\boldsymbol{\rho}}^{(j)}\right) \\
& +\left(\frac{k_{\text {in }}}{f_{\text {in }}} \frac{\exp \left(P u_{\text {out }}\left(\overline{\boldsymbol{\rho}}^{(j)}\right)\right)}{\exp \left(P u_{\text {out }}^{K S}\right)} \boldsymbol{\Lambda}^{T}+\sum_{k=1}^{N_{k}} h_{k}^{(j)} \frac{f_{\sigma}\left(\bar{\rho}_{k}^{(j)}\right)}{\hat{\sigma}_{e q}^{(k)}\left(\overline{\boldsymbol{\rho}}^{(j)}\right)}\left(\mathbf{a}_{k}^{(j)}\right)^{T} \mathbf{H}_{k}+\boldsymbol{\lambda}_{(j)}^{T} \mathbf{K}\left(\overline{\boldsymbol{\rho}}^{(j)}\right)\right) \frac{\partial \mathbf{U}\left(\overline{\boldsymbol{\rho}}^{(j)}\right)}{\partial \bar{\rho}_{n}^{(j)}}
\end{aligned}
$$

where $h_{k}^{(j)}$ and $\left(\mathbf{a}_{k}^{(j)}\right)^{T}$ are auxiliary terms, employed to obtain a more compact notation, defined as

$$
h_{k}^{(j)}=\left\langle\mu_{k}^{(j)}+r\left(\frac{\sigma_{e q}^{(k)}\left(\overline{\boldsymbol{\rho}}^{(j)}\right)}{\sigma_{y}}-1\right)\right\rangle \frac{1}{\sigma_{y}}
$$


and

$$
\left(\mathbf{a}_{k}^{(j)}\right)^{T}=\mathbf{u}_{k}^{T}\left(\overline{\boldsymbol{\rho}}^{(j)}\right) \mathbf{B}_{k}^{T} \mathbf{C}^{0} \mathbf{M} \mathbf{C}^{0} \mathbf{B}_{k}
$$

$\hat{\sigma}_{e q}^{(k)}\left(\overline{\boldsymbol{\rho}}^{(j)}\right)$ is the solid von Mises stress at point $k$, computed as

$$
\hat{\sigma}_{e q}^{(k)}\left(\overline{\boldsymbol{\rho}}^{(j)}\right)=\sqrt{\hat{\boldsymbol{\sigma}}_{k}^{T}\left(\overline{\boldsymbol{\rho}}^{(j)}\right) \mathbf{M} \hat{\boldsymbol{\sigma}}_{k}\left(\overline{\boldsymbol{\rho}}^{(j)}\right)+\sigma_{\min }^{2}} ;
$$

and $\mathbf{H}_{k}$ is a localization operator, which relates the local displacement vector to the global displacement vector, as $\mathbf{u}_{k}\left(\overline{\boldsymbol{\rho}}^{(j)}\right)=\mathbf{H}_{k} \mathbf{U}\left(\overline{\boldsymbol{\rho}}^{(j)}\right)$.

In order to avoid the computation of the derivative of $\mathbf{U}\left(\overline{\boldsymbol{\rho}}^{(j)}\right)$ with respect to $\bar{\rho}_{n}^{(j)}$, the adjoint vector $\boldsymbol{\lambda}_{(j)}$ is computed as

$$
\mathbf{K}\left(\overline{\boldsymbol{\rho}}^{(j)}\right) \boldsymbol{\lambda}_{(j)}=-\frac{k_{\text {in }}}{f_{\text {in }}} \frac{\exp \left(P u_{\text {out }}\left(\overline{\boldsymbol{\rho}}^{(j)}\right)\right)}{\exp \left(P u_{\text {out }}^{K S}\right)} \boldsymbol{\Lambda}-\sum_{k=1}^{N_{k}} h_{k}^{(j)} \frac{f_{\sigma}\left(\bar{\rho}_{k}^{(j)}\right)}{\hat{\sigma}_{\text {eq }}^{(k)}\left(\overline{\boldsymbol{\rho}}^{(j)}\right)} \mathbf{H}_{k}^{T} \mathbf{a}_{k}^{(j)} .
$$

After computing the adjoint vector $\lambda_{(j)}$, the derivative of $L_{u \sigma}$ is computed as

$$
\frac{\partial L_{u \sigma}}{\partial \bar{\rho}_{n}^{(j)}}=h_{n}^{(j)} \frac{\partial f_{\sigma}\left(\bar{\rho}_{n}^{(j)}\right)}{\partial \bar{\rho}_{n}^{(j)}} \hat{\sigma}_{e q}^{(n)}\left(\overline{\boldsymbol{\rho}}^{(j)}\right)+\lambda_{(j, n)}^{T} \frac{\partial \mathbf{k}_{n}\left(\bar{\rho}_{n}^{(j)}\right)}{\partial \bar{\rho}_{n}^{(j)}} \mathbf{u}_{n}\left(\overline{\boldsymbol{\rho}}^{(j)}\right)
$$

since the derivative of $f_{\sigma}\left(\bar{\rho}_{k}^{(j)}\right)$ is only different from zero for $\bar{\rho}_{k}^{(j)}=\bar{\rho}_{n}^{(j)}$, and the term associated with the derivative of $\mathbf{K}\left(\overline{\boldsymbol{\rho}}^{(j)}\right)$ can be evaluated through local computations, where $\lambda_{(j, n)}=\mathbf{H}_{k} \boldsymbol{\lambda}_{(j)}$.

The derivative of the augmented Lagrangian function $L$ with respect to a design variable $\rho_{m}$ is then obtained through the chain rule shown in Equation (.4), after computing the derivatives in Equations (.5), (.6), (.7) and (.13).

Note that three adjoint problems, Equation (.12), are solved to evaluate Equation (.13) for $j \in\{e, i, d\}$, i.e., one adjoint problem per physical density field.

\section{Acknowledgements}

G. A. da Silva and A. T. Beck kindly acknowledge financial support of this research project by the agencies $\mathrm{CNPq}$ (National Council for Research and Development), grant number 306373/2016-5, and FAPESP (São Paulo Research Foundation), grant number 2018/16701-1. This study was financed in part by the Coordination for the Improvement of Higher Education Personnel - Brazil (CAPES) - Finance Code 001. O. Sigmund was supported by the Villum Investigator Project InnoTop funded by the Villum Foundation. 


\section{References}

[1] P. Duysinx, M. P. Bendsøe, Topology optimization of continuum structures with local stress constraints, International Journal for Numerical Methods in Engineering 43 (8) (1998) 1453 - 1478 (1998). doi:10.1002/(SICI)1097-0207(19981230)43:8;1453::AID-NME480;3.0.CO;2-2.

[2] D. M. De Leon, J. Alexandersen, J. S. O. Fonseca, O. Sigmund, Stress-constrained topology optimization for compliant mechanism design, Structural and Multidisciplinary Optimization 52 (5) (2015) 929 - 943 (2015). doi:10.1007/s00158-015-1279-z.

[3] C. G. Lopes, A. A. Novotny, Topology design of compliant mechanisms with stress constraints based on the topological derivative concept, Structural and Multidisciplinary Optimization 54 (4) (2016) 737 - 746 (2016). doi:10.1007/s00158-016-1436-Z.

[4] S. Chu, L. Gao, M. Xiao, Z. Luo, H. Li, Stress-based multi-material topology optimization of compliant mechanisms, International Journal for Numerical Methods in Engineering 113 (7) (2018) 1021 - 1044 (2018). doi:10.1002/nme.5697.

[5] A. de Assis Pereira, E. L. Cardoso, On the influence of local and global stress constraint and filtering radius on the design of hinge-free compliant mechanisms, Structural and Multidisciplinary Optimization 58 (2) (2018) 641 - 655 (2018). doi:10.1007/s00158-018-1915-5.

[6] C. Conlan-Smith, K. A. James, A stress-based topology optimization method for heterogeneous structures, Structural and Multidisciplinary Optimization (2019). doi:10.1007/s00158-019-02207-9.

[7] O. Sigmund, Manufacturing tolerant topology optimization, Acta Mechanica Sinica 25 (2) (2009) 227-239 (Apr 2009). doi:10.1007/s10409009-0240-z.

[8] F. Wang, B. S. Lazarov, O. Sigmund, On projection methods, convergence and robust formulations in topology optimization, Structural and Multidisciplinary Optimization 43 (6) (2011) 767-784 (Jun 2011). doi:10.1007/s00158-010-0602-y.

[9] S. Chen, W. Chen, A new level-set based approach to shape and topology optimization under geometric uncertainty, Structural and Multidisciplinary Optimization 44 (1) (2011) 1 - 18 (2011). doi:10.1007/s00158-011-0660-9.

[10] F. Wang, J. S. Jensen, O. Sigmund, Robust topology optimization of photonic crystal waveguides with tailored dispersion properties, Journal of the Optical Society of America B 28 (3) (2011) 387 - 397 (2011). doi:10.1364/JOSAB.28.000387.

[11] M. Schevenels, B. Lazarov, O. Sigmund, Robust topology optimization accounting for spatially varying manufacturing errors, Computer Methods in Applied Mechanics and Engineering 200 (49) (2011) 3613 - 3627 (2011). doi:10.1016/j.cma.2011.08.006.

[12] B. S. Lazarov, M. Schevenels, O. Sigmund, Robust design of large-displacement compliant mechanisms, Mechanical Sciences 2 (2) (2011) 175 - 182 (2011). doi:10.5194/ms-2-175-2011.

[13] B. S. Lazarov, M. Schevenels, O. Sigmund, Topology optimization with geometric uncertainties by perturbation techniques, International Journal for Numerical Methods in Engineering 90 (11) (2012) 1321 - 1336 (2012). doi:10.1002/nme.3361.

[14] B. S. Lazarov, M. Schevenels, O. Sigmund, Topology optimization considering material and geometric uncertainties using stochastic collocation methods, Structural and Multidisciplinary Optimization 46 (4) (2012) 597 - 612 (2012). doi:10.1007/s00158-012-0791-7.

[15] X. Guo, W. Zhang, L. Zhang, Robust structural topology optimization considering boundary uncertainties, Computer Methods in Applied Mechanics and Engineering 253 (2013) 356 - 368 (2013). doi:10.1016/j.cma.2012.09.005.

[16] W. Zhang, Z. Kang, Robust shape and topology optimization considering geometric uncertainties with stochastic level set perturbation, International Journal for Numerical Methods in Engineering 110 (1) (2017) 31 - 56 (2017). doi:10.1002/nme.5344.

[17] G. A. da Silva, A. T. Beck, O. Sigmund, Stress-constrained topology optimization considering uniform manufacturing uncertainties, Computer Methods in Applied Mechanics and Engineering 344 (2019) 512 - 537 (2019). doi:10.1016/j.cma.2018.10.020.

[18] M. Jansen, G. Lombaert, M. Diehl, B. S. Lazarov, O. Sigmund, M. Schevenels, Robust topology optimization accounting for misplacement of material, Structural and Multidisciplinary Optimization 47 (3) (2013) 317-333 (2013). doi:10.1007/s00158-012-0835-z.

[19] M. Jansen, B. S. Lazarov, M. Schevenels, O. Sigmund, On the similarities between micro/nano lithography and topology optimization projection methods, Structural and Multidisciplinary Optimization 48 (4) (2013) 717-730 (2013). doi:10.1007/s00158-013-0941-6. 
[20] M. Zhou, B. S. Lazarov, O. Sigmund, Topology optimization for optical projection lithography with manufacturing uncertainties, Applied Optics 53 (12) (2014) 2720-2729 (2014). doi:10.1364/AO.53.002720.

[21] M. Zhou, B. S. Lazarov, O. Sigmund, Topology optimization for optical microlithography with partially coherent illumination, International Journal for Numerical Methods in Engineering 109 (5) (2017) 631-647 (2017). doi:10.1002/nme.5299.

[22] O. Sigmund, K. Maute, Topology optimization approaches, Structural and Multidisciplinary Optimization 48 (6) (2013) 1031 - 1055 (2013). doi:10.1007/s00158-013-0978-6.

[23] K.-J. Bathe, Finite Element Procedures, Prentice Hall, Upper Sadle River, New Jersey, 1996 (1996).

[24] M. P. Bendsøe, Optimal shape design as a material distribution problem, Structural optimization 1 (4) (1989) 193-202 (1989). doi:10.1007/BF01650949

[25] O. Sigmund, Morphology-based black and white filters for topology optimization, Structural and Multidisciplinary Optimization 33 (4-5) (2007) 401 - 424 (2007). doi:10.1007/s00158-006-0087-x.

[26] J. K. Guest, J. H. Prévost, T. Belytschko, Achieving minimum length scale in topology optimization using nodal design variables and projection functions, International Journal for Numerical Methods in Engineering 61 (2) (2004) 238 - 254 (2004). doi:10.1002/nme.1064.

[27] G. D. Cheng, X. Guo, ع-relaxed approach in structural topology optimization, Structural Optimization 13 (4) (1997) 258 - 266 (1997). doi:10.1007/BF01197454

[28] P. Duysinx, O. Sigmund, New developments in handling stress constraints in optimal material distribution, in: 7th AIAA/USAF/NASA/ISSMO Symposium on Multidisciplinary Analysis and Optimization, 1998, pp. 1501 - 1509 (1998). doi:10.2514/6.1998-4906.

[29] C. Le, J. Norato, T. Bruns, C. Ha, D. Tortorelli, Stress-based topology optimization for continua, Structural and Multidisciplinary Optimization 41 (4) (2010) 605 - 620 (2010). doi:10.1007/s00158-009-0440-y.

[30] E. Holmberg, B. Torstenfelt, A. Klarbring, Stress constrained topology optimization, Structural and Multidisciplinary Optimization 48 (1) (2013) 33-47 (2013). doi:10.1007/s00158-012-0880-7.

[31] M. Bruggi, P. Duysinx, Topology optimization for minimum weight with compliance and stress constraints, Structural and Multidisciplinary Optimization 46 (3) (2012) 369-384 (2012). doi:10.1007/s00158-012-0759-7.

[32] J. T. Pereira, E. A. Fancello, C. S. Barcellos, Topology optimization of continuum structures with material failure constraints, Structural and Multidisciplinary Optimization 26 (1-2) (2004) 50 - 66 (2004). doi:10.1007/s00158-003-0301-z.

[33] E. A. Fancello, Topology optimization for minimum mass design considering local failure constraints and contact boundary conditions, Structural and Multidisciplinary Optimization 32 (3) (2006) 229 - 240 (2006). doi:10.1007/s00158-006-0019-9.

[34] E. G. Birgin, J. M. Martínez, Practical Augmented Lagrangian Methods for Constrained Optimization, Society for Industrial and Applied Mathematics, Philadelphia, PA, 2014 (2014). doi:10.1137/1.9781611973365.

[35] E. A. Fancello, J. T. Pereira, Structural topology optimization considering material failure constraints and multiple load conditions, Latin American Journal of Solids and Structures 1 (1) (2003) 3 - 24 (2003).

[36] H. Emmendoerfer, E. A. Fancello, A level set approach for topology optimization with local stress constraints, International Journal for Numerical Methods in Engineering 99 (2) (2014) 129 - 156 (2014). doi:10.1002/nme.4676.

[37] H. Emmendoerfer, E. A. Fancello, Topology optimization with local stress constraint based on level set evolution via reaction-diffusion, Computer Methods in Applied Mechanics and Engineering 305 (2016) 62 - 88 (2016). doi:10.1016/j.cma.2016.02.024.

[38] G. A. da Silva, E. L. Cardoso, Stress-based topology optimization of continuum structures under uncertainties, Computer Methods in Applied Mechanics and Engineering 313 (2017) 647 - 672 (2017). doi:10.1016/j.cma.2016.09.049.

[39] G. A. da Silva, A. T. Beck, E. L. Cardoso, Topology optimization of continuum structures with stress constraints and uncertainties in loading, International Journal for Numerical Methods in Engineering 113 (1) (2018) 153 - 178 (2018). doi:10.1002/nme.5607. 
[40] G. A. da Silva, A. T. Beck, Reliability-based topology optimization of continuum structures subject to local stress constraints, Structural and Multidisciplinary Optimization 57 (6) (2018) 2339 - 2355 (2018). doi:10.1007/s00158-017-1865-3.

[41] G. A. da Silva, E. L. Cardoso, A. T. Beck, Non-probabilistic robust continuum topology optimization with stress constraints, Structural and Multidisciplinary Optimization (2018). doi:10.1007/s00158-018-2122-0.

[42] H. Emmendoerfer, E. C. N. Silva, E. A. Fancello, Stress-constrained level set topology optimization for design-dependent pressure load problems, Computer Methods in Applied Mechanics and Engineering 344 (2019) 569 - 601 (2019). doi:10.1016/j.cma.2018.10.004.

[43] G. Kreisselmeier, R. Steinhauser, Systematic control design by optimizing a vector performance index, IFAC Proceedings Volumes 12 (7) (1979) 113 - 117 (1979). doi:10.1016/S1474-6670(17)65584-8.

[44] N. Olhoff, Multicriterion structural optimization via bound formulation and mathematical programming, Structural optimization 1 (1) (1989) 11 - 17 (1989). doi:10.1007/BF01743805.

[45] S. S. Rao, Engineering Optimization: Theory and Practice, 4th Edition, Wiley, New Jersey, 2009 (2009).

[46] O. Sigmund, On the design of compliant mechanisms using topology optimization, Mechanics of Structures and Machines 25 (4) (1997) 493 524 (1997). doi:10.1080/08905459708945415.

[47] A. Clausen, E. Andreassen, On filter boundary conditions in topology optimization, Structural and Multidisciplinary Optimization 56 (5) (2017) 1147-1155 (2017). doi:10.1007/s00158-017-1709-1.

[48] G. Christophe, R. Jean-Francois, Gmsh: A 3-d finite element mesh generator with built-in pre- and post-processing facilities, International Journal for Numerical Methods in Engineering 79 (11) (2009) 1309-1331 (2009). doi:10.1002/nme.2579. 